ReVISTA de BIOLOGía TROPICAL

\title{
Ecosistemas coralinos del Parque Nacional Isla del Coco, Costa Rica: estructura y comparación 1987-2014
}

\author{
Juan José Alvarado1,2,3, Andrés Beita-Jiménez ${ }^{2}$, Sebastián Mena², Cindy Fernández-García ${ }^{1,2,4}$, \\ Ana Gloria Guzman-Mora ${ }^{5} \&$ Jorge Cortés $1,2,3$ \\ 1. Centro de Investigación en Ciencias del Mar y Limnología (CIMAR), Universidad de Costa Rica (UCR), San Pedro, \\ 11501-2060 San José, Costa Rica; juanalva76@yahoo.com, cindyfdez@yahoo.com, jorge.cortes@ucr.ac.cr \\ 2. Escuela de Biología, Universidad de Costa Rica; abeitaj@gmail.com, sebasmenago@gmail.com \\ 3. Museo de Zoología, Universidad de Costa Rica. \\ 4. Herbario de la Universidad de Costa Rica (USJ). \\ 5. Conservación Internacional; aguzman@conservation.org
}

\section{Recibido 17-IV-2015. Corregido 22-VIII-2015. Aceptado 31-VIII-2015.}

\begin{abstract}
Coral reefs of Isla del Coco National Park, Costa Rica: structure and comparison (1987-2014). Isla del Coco has one of the most diverse and well-conserved coral reefs in Costa Rica. These reefs have been extensively studied since the 1980's, however those studies focused mainly on coral coverage. The aim of this study is to evaluate the coral reefs of the island, not only by the coverage of the substrate but also the composition of fish, invertebrates and reef complexity. A total of 17 sites around the island were sampled in July 2013 and in February-March 2014. At each site, we evaluated three depths (4-8 m, 9-12 m and 13-16 m), where three $10 \mathrm{~m}$ long transects were sampled. Turf algae was the predominant substrate cover with $38.18 \pm 5.58 \%$. The crustose calcareous algae (Order Corallinales) had a $28.12 \pm 5.85 \%$ cover and live coral was $18.64 \pm 3.55$ $\%$, with Porites lobata as the main reef builder. A total of 18 taxa of macroinvertebrates were observed in the study sites, of which $56 \%$ of the species were not abundant, $33 \%$ were common, and the sea urchin Diadema mexicanum was the only dominant species. The density of lobsters of the genus Panulirus was $388 \pm 385$ ind $\mathrm{ha}^{-1}$, which is the highest density reported in the Eastern Tropical Pacific. Also we observed high densities of the sea cucumber Isostichopus fuscus (550 ind ha ${ }^{-1}$ ), which has a high commercial value. We recorded 45338 fish individuals, distributed in 93 species, with an average of $1.2 \pm 8.5 \mathrm{tn} \mathrm{ha}^{-1}$. From the total of species, $51 \%$ were uncommon, $31 \%$ common, $11 \%$ predominant and $8 \%$ occasional. The fish community at Isla del Coco is represented by a large number of carnivorous species, followed by top predators, herbivores and planktivorous species. According to the fish categories of the IUCN Red List, $16 \%$ of species are in threatened categories and represent $25 \%$ of the total sampled biomass. When comparing with studies carried out from 1987 to the present, the recovery in coral cover over time is evident, mainly due to: 1) a decrease in the pressure of the bioerosive action of D. mexicanum; and 2) the conservation actions that have been taken on the island. This has been translated in complex and more rugose reefs. Isla del Coco reefs are healthy, with functional and complex food webs; where species of high commercial value have large populations. The effectiveness of conservation and management activities in Isla del Coco has resulted in the recovery of the reef communities. Rev. Biol. Trop. 64 (Suppl. 1): S153-S175. Epub 2016 Febrary 01.
\end{abstract}

Key words: live coral cover, Diadema mexicanum, recovery, resilience, biomass, conservation.

La Isla del Coco, ubicada a más de 500km de la costa Pacífica de Costa Rica, era conocida hasta inicios de la década de 1970, como un sitio de paso de barcos balleneros, pescadores y buscadores de tesoros (Weston-Knight, 1992; Cortés, 2008a). Estos visitantes llegaban a la isla a saquear sus recursos terrestres y marinos. Los pescadores podían quedarse días pescando en las ricas aguas sin ningún control de sus capturas. A finales de la década de 1970, se reconoce la riqueza terrestre y marina de la isla y se declara Parque Nacional. Ya para esa fecha 
los fondos marinos se veían afectados por la presencia humana (J. Cortés, obs. pers.), con restos de basura en el fondo, aceite de barco en el agua y un impacto sobre las poblaciones de peces.

El proceso de conservación de la Isla ha sido lento, lleno de obstáculos e inconvenientes tanto humanos como naturales. En 1978, se declara como Parque Nacional, el cual incluye la isla y todos sus islotes (Decreto Ejecutivo 8748 del 22 junio de 1978). En esta declaratoria se deja de lado la parte marina, y no es hasta 1984 que se incluyen $5 \mathrm{~km}$ alrededor de la isla como área protegida. Dos de los argumentos que justifican esta ampliación fueron primero, que la isla posee un ambiente marino extremadamente diverso con presencia de especies endémicas, y segundo la alta diversidad de la fauna marina asociada a las formaciones coralinas (Decreto Ejecutivo 15514 del 14 junio de 1984). En 1991, esta área marina protegida se amplío hasta $15 \mathrm{~km}$ alrededor de la isla, con base en la gran afluencia y diversidad de la fauna marina que existía en los alrededores de la misma, la cual fue sobreexplotada, y se encuentraba en peligro de extinción, como el tiburón martillo, entre otras especies (Decreto Ejecutivo 20260 del 09 enero de 1991). En 2001 se da la última ampliación hasta $22.2 \mathrm{~km}$. Esta ampliación estuvo sustentada en la conservación de especies migratorias, de alto valor comercial y en vías de extinción que utilizan las aguas circundantes de la isla como sitio de crianza, protegiendo de esta manera parte de la Cordillera Submarina del Coco, que es utilizada como ruta migratoria. Así mismo, otro argumento fuerte para esta ampliación estuvo basado en el reconocimiento por parte del Gobierno de que esta zona es una de las áreas que más intensamente han sido explotadas por flotas pesqueras nacionales e internacionales, donde los controles han sido deficientes (Decreto Ejecutivo 29834 del 23 de agosto del 2001). Con el establecimiento en el 2011 del Área Marina de Manejo Montes Submarinos (AMMMS), se establece un área de amortiguamiento alrededor del Parque Nacional Isla del Coco (PNIC), dentro de la cual se prohíbe la pesca mediante redes de arrastre, la pesca semi-industrial e industrial y cualquier tipo de exploración y explotación petrolera (Decretos Ejecutivos 35369 del 18 de mayo del 2009 y 36452 del 3 de marzo del 2011). Esta nueva área marina protegida implica que el área protegida alrededor de la isla se amplié entre 38 y $120 \mathrm{~km}$ alrededor de la isla, dependiendo del sector, debido a la forma del AMMMS.

El resultado de la perseverancia en conservar dicha isla, ha permitido una recuperación envidiable de sus ecosistemas marinos (Guzman, \& Cortés, 2007; Friedlander et al., 2012). Recientes evaluaciones que comparan siete áreas marinas protegidas (AMPs) de Costa Rica, Panamá, Colombia y Ecuador, clasificándolas en tres categorías (zonas de alta protección, zonas de limitada protección y zonas de pescas), indican el alto valor en términos de conservación que posee la Isla del Coco (Edgar et al., 2011). Esta isla fue clasificada como una zona de alta protección, y en comparación con las otras zonas, resultó poseer cinco veces más biomasa de peces carnívoros. En este sentido, fue más evidente el efecto de la pesca sobre las AMPs continentales que en las oceánicas como la Isla del Coco. Así mismo, posee una mayor cobertura de coral vivo y de algas calcáreas costrosas, y una mayor abundancia de erizos de mar y langostas en comparación con las otras AMPs (Edgar et al., 2011). Parte del estado de conservación de la isla se debe principalmente a cinco factores: 1) grado de pesca permitido dentro del área protegida, 2) nivel de protección, 3) edad del AMP (>10 años), tamaño del AMP $\left(>100 \mathrm{~km}^{2}\right)$, y 5) nivel de aislamiento (aislada por aguas profundas) (Edgar et al., 2014). Estos cinco criterios fueron aplicados a 87 AMPs a nivel global, y solo cuatro lograron cumplir con los cinco criterios, entre ellas el Parque Nacional Isla del Coco.

Con este trabajo se pretende demostrar los efectos positivos de las estrategias de conservación en el PNIC a través del tiempo, y como ellas han facilitado una recuperación de los ecosistemas arrecifales, y de las poblaciones de organismos que en ellas habitan, aumentando su resiliencia. Este trabajo elabora 
una línea base, mediante la utilización de una metodología estándar, del estado actual (estructura y composición) de los arrecifes del PNIC. Además, se realiza una comparación temporal de estos sistemas con investigaciones previas para determinar tendencias de cambio en el estado de conservación de los recursos marinos que permitan identificar recomendaciones para su manejo.

\section{MATERIALES Y MÉTODOS}

Sitio de estudio: El Parque Nacional Isla del Coco (PNIC) consiste de una isla principal de $24 \mathrm{~km}^{2}$ y varias islas más pequeñas e islotes alrededor sobre una plataforma insular (Lizano, 2008; 2012). El área marina protegida es de casi $2000 \mathrm{~km}^{2}$ (Alvarado, Cortés, Esquivel, \& Salas, 2012). Una amplia descripción de las condiciones oceanográficas y biológicas pueden ser encontradas en Cortés (2008b; 2012).

Metodología de campo: Dentro de PNIC se visitaron 17 sitios (Cuadro 1). Se realizaron dos visitas, del 12 al 20 de julio 2013 y del 23 de febrero al 3 de marzo 2014. En cada sitio, se trabajaron tres profundidades (somero 4-8 m, intermedio 9-12 m y profundo 13-16 m). En cada profundidad se definieron, mediante una cinta métrica, tres transectos de $10 \mathrm{~m}$ de longitud, paralelos a la costa y separados por $10 \mathrm{~m}$ entre ellos. En cada transecto se cuantificó: 1) cobertura de fondo y rugosidad del arrecife, 2) la diversidad y densidad de macroinvertebrados y 3) la diversidad y biomasa de peces arrecifales.

Cobertura del fondo y rugosidad: En cada uno de los transectos, se colocó una cuadrícula de $1 \mathrm{~m}^{2}$, subdividida en cuadrantes de $0.01 \mathrm{~m}^{2}$, para analizar la cobertura del fondo (Weinberg, 1981). La cobertura se cuantificó en los diez metros consecutivos a lo largo de cada transecto, siguiendo la cinta métrica sin dejar espacios entre ellos. De esta forma, se analizaron tres transectos $(10 \mathrm{~m}$ largo $\mathrm{x} 1 \mathrm{~m}$ ancho) en cada profundidad. Se definieron las siguientes categorías para describir la cobertura

CUADRO 1

Sitios de muestreo de los ecosistemas arrecifales en el Parque Nacional Isla del Coco

TABLE 1

Sampled sites with presence of reef ecosystems at Isla del Coco National Park

\begin{tabular}{|c|c|c|c|c|c|}
\hline \# & Sitio & Coordenadas & \# transectos & Ubicación & Exposición \\
\hline 1 & Bahía Chatham & $5^{\circ} 33^{\prime} 9.20^{\prime \prime} \mathrm{N}-87^{\circ} 2^{\prime} 27.50^{\prime \prime} \mathrm{W}$ & 6 & norte & no \\
\hline 2 & Bahía Wafer & $5^{\circ} 32^{\prime} 45.40^{\prime \prime} \mathrm{N}-87^{\circ} 3 ’ 44.30^{\prime \prime} \mathrm{W}$ & 12 & norte & no \\
\hline 3 & Bahía Weston & $5^{\circ} 33^{\prime} 8.20^{\prime \prime} \mathrm{N}-87^{\circ} 3^{\prime} 3.20^{\prime \prime} \mathrm{W}$ & 14 & norte & no \\
\hline 4 & Bahía Yglesias & $5^{\circ} 30^{\prime} 20.80^{\prime \prime} \mathrm{N}-87^{\circ} 4^{\prime} 3.90^{\prime \prime} \mathrm{W}$ & 6 & sur & si \\
\hline 5 & Dos Amigos Pequeño & $5^{\circ} 30^{\prime} 30.80^{\prime \prime} \mathrm{N}-87^{\circ} 6^{\prime} 4.40^{\prime \prime} \mathrm{W}$ & 3 & sur & si \\
\hline 6 & Isla Manuelita Adentro & $5^{\circ} 33^{\prime} 42.70^{\prime \prime} \mathrm{N}-87^{\circ} 2^{\prime} 48.90^{\prime \prime} \mathrm{W}$ & 12 & norte & no \\
\hline 7 & Isla Manuelita Afuera & $5^{\circ} 33^{\prime} 39.40^{\prime \prime} \mathrm{N}-87^{\circ} 2 ’ 54.60^{\prime \prime} \mathrm{W}$ & 3 & norte & si \\
\hline 8 & Isla Pájara & $5^{\circ} 33^{\prime} 14.80^{\prime \prime} \mathrm{N}-87^{\circ} 3^{\prime} 14.70^{\prime \prime} \mathrm{W}$ & 15 & norte & no \\
\hline 9 & Isla Ulloa & $5^{\circ} 33^{\prime} 5.20^{\prime \prime} \mathrm{N}-87^{\circ} 1^{\prime} 56.80^{\prime \prime} \mathrm{W}$ & 6 & norte & no \\
\hline 10 & Isla Vikinga & $5^{\circ} 32^{\prime} 58.40^{\prime \prime} \mathrm{N}-87^{\circ} 3{ }^{\prime} 52.20^{\prime \prime} \mathrm{W}$ & 12 & norte & no \\
\hline 11 & Punta Gissler & $5^{\circ} 32^{\prime} 36.10^{\prime \prime} \mathrm{N}-87^{\circ} 4^{\prime} 18.30^{\prime \prime} \mathrm{W}$ & 15 & norte & si \\
\hline 12 & Punta Leonel & $5^{\circ} 31^{\prime} 9.50^{\prime \prime} \mathrm{N}-87^{\circ} 5^{\prime} 44.90^{\prime \prime} \mathrm{W}$ & 15 & sur & si \\
\hline 13 & Punta María & $5^{\circ} 32^{\prime} 7.30^{\prime \prime} \mathrm{N}-87^{\circ} 5^{\prime} 12.50^{\prime \prime} \mathrm{W}$ & 12 & norte & si \\
\hline 14 & Punta Presidio & $5^{\circ} 32^{\prime} 59.80^{\prime \prime} \mathrm{N}-87^{\circ} 3{ }^{\prime} 37.40^{\prime \prime} \mathrm{W}$ & 15 & norte & no \\
\hline 15 & Roca Sucia & $5^{\circ} 32^{\prime} 53.60^{\prime \prime} \mathrm{N}-87^{\circ} 4^{\prime} 54.10^{\prime \prime} \mathrm{W}$ & 3 & norte & si \\
\hline 16 & Roca Sumergida & $5^{\circ} 30^{\prime} 17.50^{\prime \prime} \mathrm{N}-87^{\circ} 3 ’ 19.60^{\prime \prime} \mathrm{W}$ & 4 & sur & si \\
\hline 17 & Rodolitos & $5^{\circ} 32^{\prime} 45.30^{\prime \prime} \mathrm{N}-87^{\circ} 1^{\prime} 44.70^{\prime \prime} \mathrm{W}$ & 6 & norte & si \\
\hline
\end{tabular}


del sustrato: coral vivo (a nivel de especie), coral muerto, coral blanqueado, macroalgas (a nivel de género, incluyendo algas foliosas y carnosas), tapete algal (TA) (algas filamentosas y corticadas de pocos milímetro de alto formando una alfombra densa), algas calcáreas costrosas (ACC) del Orden Corallinales, otras algas costrosas (AC), esponja, anémonas, arena, cascajo y otros organismos (e.g. briozoarios, hidrozoos, zoántidos, cirripedios, tunicados, cianobacterias).

La complejidad arrecifal se analizó mediante la medición del índice de rugosidad (IR) del sustrato, variable relacionada con la diversidad, abundancia y estado de salud de los organismos presentes en los arrecifes (ÁlvarezFilip, Côté, Gill, Watkinson, \& Dulvy, 2011; Graham, Jennings, MacNeil, Mouillot, \& Wilson, 2015). Para determinar esta variable, en cada transecto, se extendió una cadena de $10 \mathrm{~m}$ de largo con eslabones de $1 \mathrm{~cm}$, siguiendo el contorno del fondo a lo largo de cada uno de los transectos. Mediante la cinta métrica, se estimó la distancia total en línea recta del punto de inicio al punto final que alcanzó la cadena (Rogers, Garrison, Grober, Hillis, \& Franke, 1994; Lee, 2006). El IR se calcula restándole a 1 la razón entre la distancia recorrida por la cadena sobre el fondo y la distancia total de la cadena. Para este índice valores cercanos a 0 representan arrecifes planos, mientras que valores cercanos a 1 representan arrecifes rugosos o más complejos morfológicamente.

Diversidad y abundancia de macroinvertebrados: Se muestreó $1 \mathrm{~m}$ a ambos lados de la línea de cada uno de los transectos establecidos $\left(10 \mathrm{~m} \times 2 \mathrm{~m}=20 \mathrm{~m}^{2}\right)$. El buzo se enfocó en revisar entre grietas y rocas, sin causar alguna alteración del sustrato. Los macroinvertebrados contabilizados fueron animales conspicuos de más de $2.5 \mathrm{~cm}$. Las categorías fueron moluscos (bivalvos, cefalópodos y gasterópodos, principalmente opistobranquios), crustáceos (langostas y cangrejos flecha) y equinodermos (pepinos, erizos y estrellas de mar). Se identificaron los organismos a nivel de género y especie en la medida de lo posible.
Diversidad y biomasa de peces arrecifales: Para determinar la composición íctica en el arrecife, se contabilizó y se estimó la talla de todos los peces observados de la línea del transecto a $5 \mathrm{~m}$ hacia un lado (ancho) y $5 \mathrm{~m}$ de alto $(10 \mathrm{mx} 5 \mathrm{mx} 5 \mathrm{~m})$, formando un túnel imaginario. Se estimó el tamaño de cada pez y se clasificaron en las siguientes categorías: $<5,5-10,10-15,15-20,20-25,25-50,50-100$, $100-150,150-200,200-250,250-300 \mathrm{~cm}$. Las frecuencias de tallas de cada especie se transformaron en biomasa usando los parámetros $a$ y $b$ obtenidos de FishBase, donde:

\section{Peso $=a \times(\text { longitud total })^{\mathrm{b}}$}

Las especies se agruparon en categorías de abundancia con base en la densidad (ind $\mathrm{m}^{-2}$ para macroinvertebrados e ind $\mathrm{ha}^{-1}$ para peces) y la presencia (número de sitios donde la especie fue observada). De esta manera, se establecieron cuatro categorías: 1) predominantes (la densidad y la presencia son mayores que el promedio), 2) raras (ambos parámetros son menores que el promedio), 3) ocasionales (la densidad supera el promedio, pero la presencia está por debajo del mismo) y 4) comunes (la presencia y la densidad están sobre y bajo el promedio respectivamente). De acuerdo a su nivel trófico, las especies se clasificaron en: depredadores tope, carnívoros, planctívoros y herbívoros sensu (Friedlander, \& DeMartini, 2002).

Análisis estadístico: Para determinar diferencias entre los sitios con respecto a la cobertura de ACC, algas costrosas, arena, coral vivo, macroalgas, TA e índice de rugosidad, entre los sitios, se realizó una serie de pruebas no paramétricas de Kruskal-Wallis, debido a que ninguna de las variables cumplió con los supuestos del ANOVA (Bakus, 2007). Todas estas pruebas estadísticas fueron realizadas en el programa Sigma Stat 3.5.

Para determinar la similitud entre las localidades, en términos de la cobertura del fondo, la densidad de macroinvertebados y la biomasa de peces, se realizó un análisis de agrupamiento 
jerárquico y un análisis de escalamiento multidimensional no métrico (nMDS) (Clarke, \& Gorley, 2006), basados en una matriz de similitud de Bray-Curtis. Las variables fueron estandarizadas y transformadas por la función raíz cuarta en el caso de los peces y por la función $\log _{10}(\mathrm{X}+1)$ en el caso de la cobertura del fondo y los macroinvertebrados.

Además, para determinar diferencias entre los agrupamientos de localidades y el grado de protección, se realizó un análisis de similitud de una vía (ANOSIM). Se dividieron los sitios con base en dos factores: 1) ubicación en la isla (parte norte o sur de la isla) y 2) grado de exposición (si: en una bahía o protegido por un islote; no: expuesto a mar abierto) (Cuadro 1). Para determinar diferencias entre los índices, según la ubicación y exposición de los sitios, se realizaron análisis de varianza de una vía en rangos (Prueba Wilcoxon). Con el fin de determinar cuál de las categorías de cobertura de sustrato está influyendo más entre los sitios, se realizó un análisis de componentes principales con la matriz de datos transformada. Con el fin de determinar cuál de las variables está contribuyendo más a la similitud entre las localidades, se realizó una serie de análisis de contribución porcentual de especie (SIMPER). Para los análisis se utilizaron los programas JMP ${ }^{\circ} 7.0$ y PRIMER $® 6.1 .4$

Para los datos de macroinvertebrados y los peces, se calculó para cada sitio los índices de diversidad de Shannon $\left(\mathrm{H}^{\prime}, \log _{10}\right)$ y la diferenciación taxonómica $\left(\Delta^{*}\right.$; Clarke, \& Warwick, 2001). Es importante aclarar que para las pruebas de Wilcoxon con $H^{\prime}\left(\log _{10}\right)$ y $\Delta^{*}$ se excluyó del análisis a Roca Sucia por presentar una sola especie. Para el $\Delta^{*}$ se utilizaron seis niveles taxonómicos: especie, género, familia, orden, clase, y filo; con sus respectivos valores (pesos) $\omega=16.66$ (especies en el mismo género), 33.33 (misma familia pero géneros diferentes), 50 (mismo orden pero familia diferente), 66.66 (misma clase pero orden diferente), 83.33 (mismo Filo pero clase diferente) y 100 (filos diferentes). Estos dos índices se compararon por localidad para determinar diferencias a través de un ANOVA de una vía. Todos estos análisis se llevaron a cabo utilizando el software PRIMER 6.0 (Clarke, \& Gorley, 2006).

\section{RESULTADOS}

Cobertura del fondo y rugosidad: En la Isla del Coco, la cobertura del fondo que predominó fue el tapete algal (TA) con 38.18 \pm 5.58 $\%$, seguida por las algas calcáreas costrosas (ACC) con $28.12 \pm 5.85 \%$ y coral vivo con $18.64 \pm 3.55 \%$ (Anexo 1). La cobertura promedio de macroalgas $(0.36 \pm 0.11 \%)$, algas costrosas (AC) $(1.69 \pm 0.42 \%)$ y rodolitos dentro del arrecife $(0.87 \pm 1.55 \%)$ fue baja. Sin embargo, en el sitio 17, la cobertura de rodolitos fue de 26.17 \%. La mayor cobertura de TA se observó en Bahía Wafer con $69.01 \%$, mientras que las mayores coberturas de ACC (65.58 \%) y coral $(55.44 \%)$ fueron en Roca Sucia e Isla Pájara, respectivamente (Anexo 1). El sustrato "Otro" que estuvo compuesto por cirripedios, briozoarios y otros organismos incrustantes, tuvo una cobertura promedio para la isla de $5.26 \pm 2.86 \%$, siendo máxima en Dos Amigos Pequeño (44.17\%), donde había una alta cobertura de cirripedios. Se observaron diferencias entre los sitios $(p<0.001)$ en cuanto a la cobertura de ACC, AC, macroalgas, TA, arena y coral (Cuadro 2).

\section{CUADRO 2}

Análisis de variancias de una vía en rangos (prueba de Kruskal-Wallis) entre los sitios de muestreo de acuerdo a la categoría de sustrato y la rugosidad en el PNIC

TABLE 2

Kruskal-Wallis One Way Analysis of Variance Rank based on substrate factor and rugosity between sampled sites at PNIC

\begin{tabular}{lccc}
\multicolumn{1}{c}{ Categoría } & $\mathrm{H}$ & $\mathrm{g} .1$. & $\mathrm{p}$ \\
Alga calcárea costrosa & 114.22 & 16 & $<0.001$ \\
Alga costrosa & 39.12 & 16 & $<0.001$ \\
Arena & 106.74 & 16 & $<0.001$ \\
Coral & 93.35 & 16 & $<0.001$ \\
Macroalga & 60.52 & 16 & $<0.001$ \\
Tapete algal & 80.38 & 16 & $<0.001$ \\
Rugosidad & 52.85 & 16 & $<0.001$ \\
\hline
\end{tabular}


Los arrecifes del PNIC resultaron complejos, con un promedio ( \pm error estándar) de $0.41 \pm 0.02$ del índice de rugosidad (IR). El IR mayor fue en Roca Sucia con 0.60, y el más baja para Dos Amigos Pequeño con 0.30 (Anexo 1). Al comparar entre todos los sitios, se observaron diferencias (Cuadro 2).

Los tapetes de algas presentaron la mayor cobertura del fondo en la Isla del Coco, estos estaban compuestos por una combinación de especies de algas rojas, verdes y pardas, las cuales variaron en composición en cada uno de los sitios. Las principales especies en los tapetes fueron: Dictyota humifusa, Cladophora, Chlorodesmis, Polysiphonia, Ceramium, Gelidium, Amphiroa y, algas pardas filamentosas del orden Ectocarpales. Las algas calcáreas costrosas pertenecen al Orden Corallinales y se encontró los géneros Lithophyllum y Lithothamnion, principalmente. En la categoría algas costrosas se encontraron varias especies de la Familia Peyssoneliaceae, dos especies del alga parda Lobophora y el alga verde Codium picturatum, las cuales fueron muy abundantes en algunos sitios. Dentro de las principales macroalgas foliosas y carnosas encontramos parches de D. humifusa, Caulerpa serrulata, Colpomenia ramosa, Digenea simplex y Padina crispata.

Los arrecifes coralinos de la Isla del Coco resultaron compuestos principalmente por el coral Porites lobata (Fig. 1), que es una especie masiva, y permite una mayor irregularidad en el fondo. Esta especie de coral fue la que presentó el mayor porcentaje de contribución a la cobertura de coral con $49.3 \%$. La segunda especie en contribución porcentual fue Psammocora profundacella con $19.0 \%$, seguido por especies del género Pocillopora con $9.3 \%$ (Fig. 1). Otras especies tuvieron una contribución inferior al $6.3 \%$. Al comparar los sitios entre sí, utilizando su ubicación en la isla, se evidenció que difierieron los sitios ubicados en el norte con los del sur de la isla (Fig. 2) (ANOSIM, $\mathrm{R}=0.45, \mathrm{p}<0.050$ ). Sin embargo, a pesar de esta diferencia, dos de los sitios del sur se agruparon con la gran mayoría de sitios del norte (Fig. 2). Tomando como patrón de

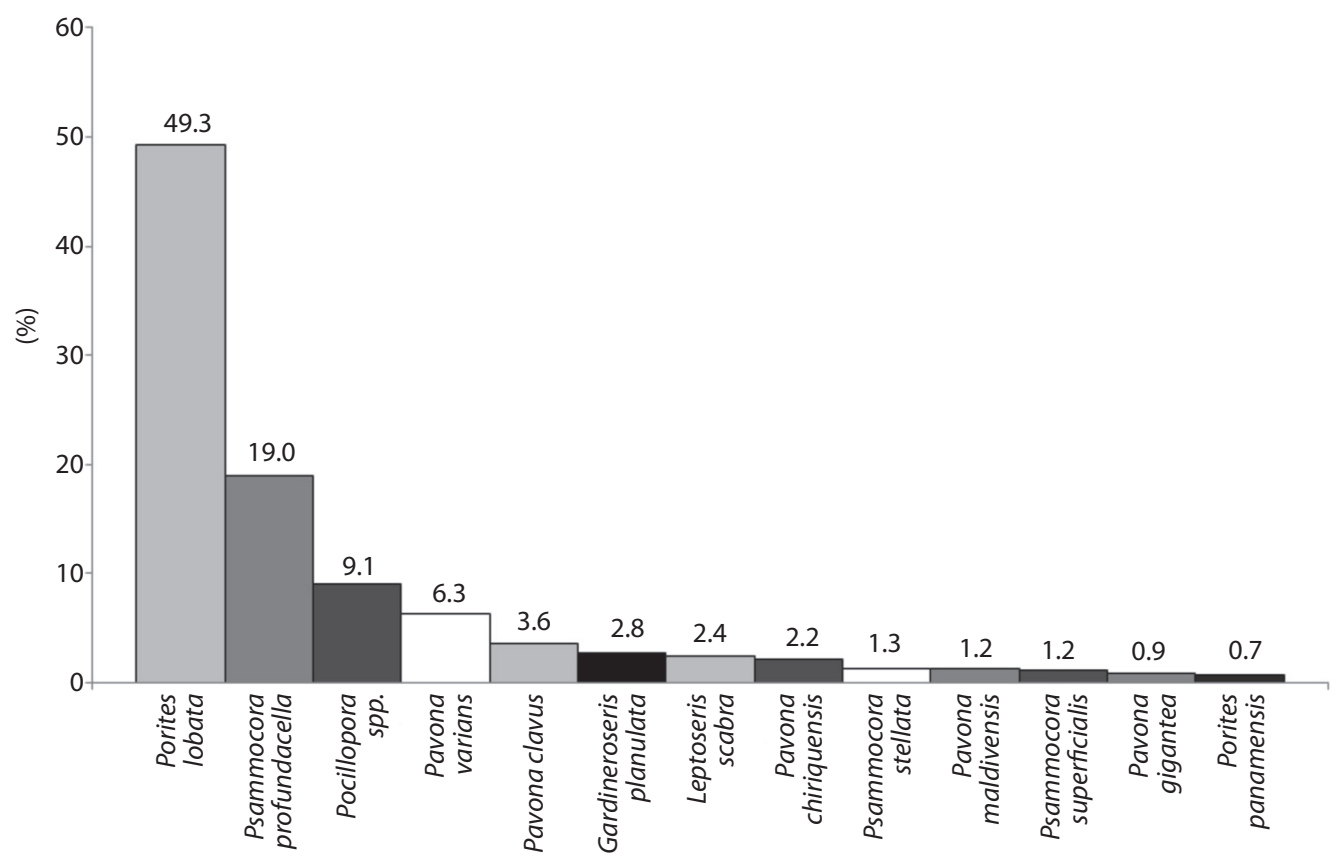

Fig. 1. Contribución porcentual por especie de coral al total de la cobertura coralina en el Parque Nacional Isla del Coco. Fig. 1. Coral species percentages of the total coral cover at Isla del Coco National Park. 


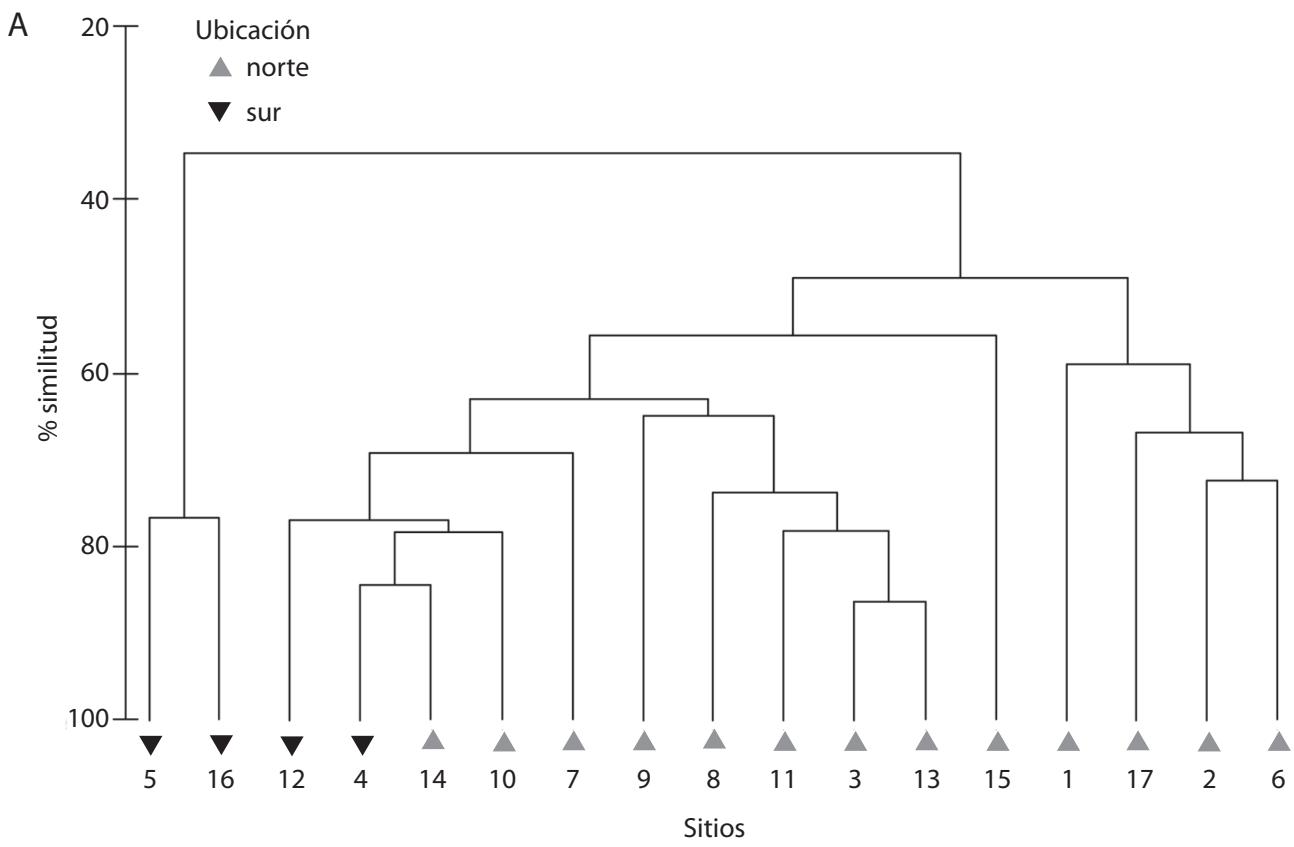

B $\quad$ Nivel de estrés $=0.1$

$\%$ de similitud

60
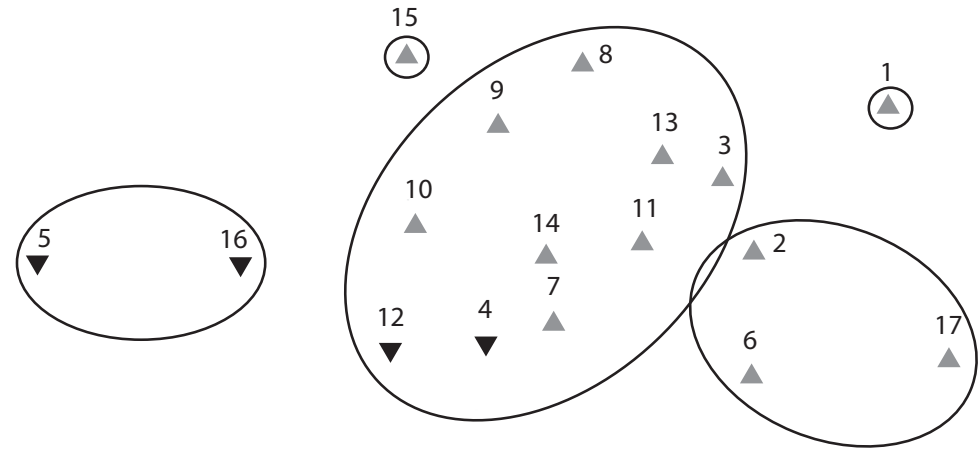

Fig. 2. (A) Dendrograma de unión simple (B) análisis multidimensional escalado por factor ubicación, basados en una matriz de similitud de Bray-Curtis utilizando los porcentajes de cobertura del sustrato en los sitios de muestreo en el Parque Nacional Isla del Coco. Número de los sitios en el Cuadro 1.

Fig. 2. (A) Hierarchical cluster analyses (B) Non metric multi-dimentional scaling analysis by location factor, based on a Bray Curtis similarity matrix using substrate cover percentages values in the sampled sites at Isla del Coco National Park. Site numbers in Table 1.

referencia un $60 \%$ de similitud entre los sitios se dieron cinco agrupaciones. Las primeras dos, fueron conformadas por un único sitio, que se separaron del resto. Bahía Chatham (\#1) contó con una baja cobertura de AAC, una moderada cobertura de coral, y la cobertura de arena más alta de todos los sitios; mientras que Roca Sucia (\#15) tuvo una cobertura moderada de ACC, coral y "Otro" sustrato. Ambos sitios no contarón con cobertura de esponjas y de 
Rodolitos (Anexo 1). La tercera agrupación la conformaron dos sitios del sur, Dos Amigos Pequeño (\#5) y Roca Sumergida (\#16), los cuales tenían en común una alta cobertura de ACC, esponjas y "Otro" sustrato, y una baja cobertura de coral (Anexo 1). La cuarta agrupación se conformó por Bahía Wafer (\#2), Isla Manuelita adentro (\#6) y Rodolitos (\#17), que comparten en común ser los únicos sitios que presentaron cobertura de rodolitos de moderada a muy alta. Los otros sitios no presentaron este tipo de categoría. Así mismo, estos sitios tuvieron coberturas bajas de AAC y "Otro", y coberturas moderadas de coral, arena y esponja. La quinta agrupación comprendió el resto de sitios evaluados, donde las coberturas de ACC y coral fueron de moderadas a altas, y las coberturas de arena, "Otro" y esponja fueron bajas.

De acuerdo al análisis de componentes principales (ACP), con tres componentes se explica el $78.6 \%$ de la variación espacial de los sitios (Fig. 3). El CP1 explica el $41.9 \%$ y está compuesto principalmente por la cobertura de esponja, "Otro" y ACC. El CP2 explica el $21.8 \%$ y está compuesto de AC, cascajo y macroalgas, mientras que el CP3 explica el $14.9 \%$ de la variación y está compuesto por la categoría coral. A los sitios del sur los está influyendo fuertemente el CP1, y la diferencia radical entre estos sitios es que a los sitios 4 y 12, al igual que a los sitios del norte, los influye fuertemente el CP2. Por su parte, a los sitios del norte lo influye fuertemente el CP3 (Fig. 3). Esta influencia se comprueba con el SIMPER (Cuadro 3), en donde el porcentaje de disimilitud entre los sitios de norte y sur fue de $56.07 \%$, y estuvo dado por la presencia de las categorías del sustrato esponja y "Otro". Por su parte, la similitud entre los sitios del norte fue de un $59.13 \%$ y estuvo explicada por las contribuciones de las categorías coral, TA y ACC. La similitud de los sitios del sur fue de un $62.96 \%$ y en ella influyeron principalmente las coberturas de esponja, ACC y "Otro" (Cuadro 3).

Diversidad y abundancia de macroinvertebrados: Se observaron 18 taxones de macroinvertebrados en los sitios estudiados (Anexo 2). En su mayoría (56 \%) fueron especies poco abundantes, $33 \%$ fueron comunes; la estrella Linckia columbiae fue la única ocasional, y el erizo de mar Diadema mexicanum fue la única especie predominante (Fig. 4).

Los sitios que presentaron mayor riqueza de especies (S) fueron Isla Vikinga (12), Isla Pájara (11), Bahía Weston (9), Punta Gissler (9) y Punta Presidio (9) (Anexo 2). El sitio con menor riqueza fue Roca Sucia con una especie (D. mexicanum). No se encontraron diferencias en la riqueza de especies respecto a la ubicación de los sitios de muestreo (Cuadro 4), sin

CUADRO 3

Análisis de Similitud porcentual de la categoría de sustrato por el factor ubicación de muestreo en el PNIC.

TABLE 3

Analysis of Similarities of substrate category between localities factor at PNIC.

\begin{tabular}{lccc} 
Ubicación & \% Similitud & Categoría de sustrato & \% Contribución \\
Norte & 59.13 & coral & 24.25 \\
& & TA & 22.40 \\
& & ACC & 12.86 \\
\hline Sur & 62.96 & Esponja & 31.99 \\
& & ACC & 23.30 \\
Ubicación & \% Disimilitud & Otro & 14.38 \\
Norte-Sur & 56.07 & Categoría de sustrato & \% Contribución \\
& & Esponja & 18.54 \\
& & Otro & 15.17 \\
& & Cascajo & 10.30 \\
\hline
\end{tabular}



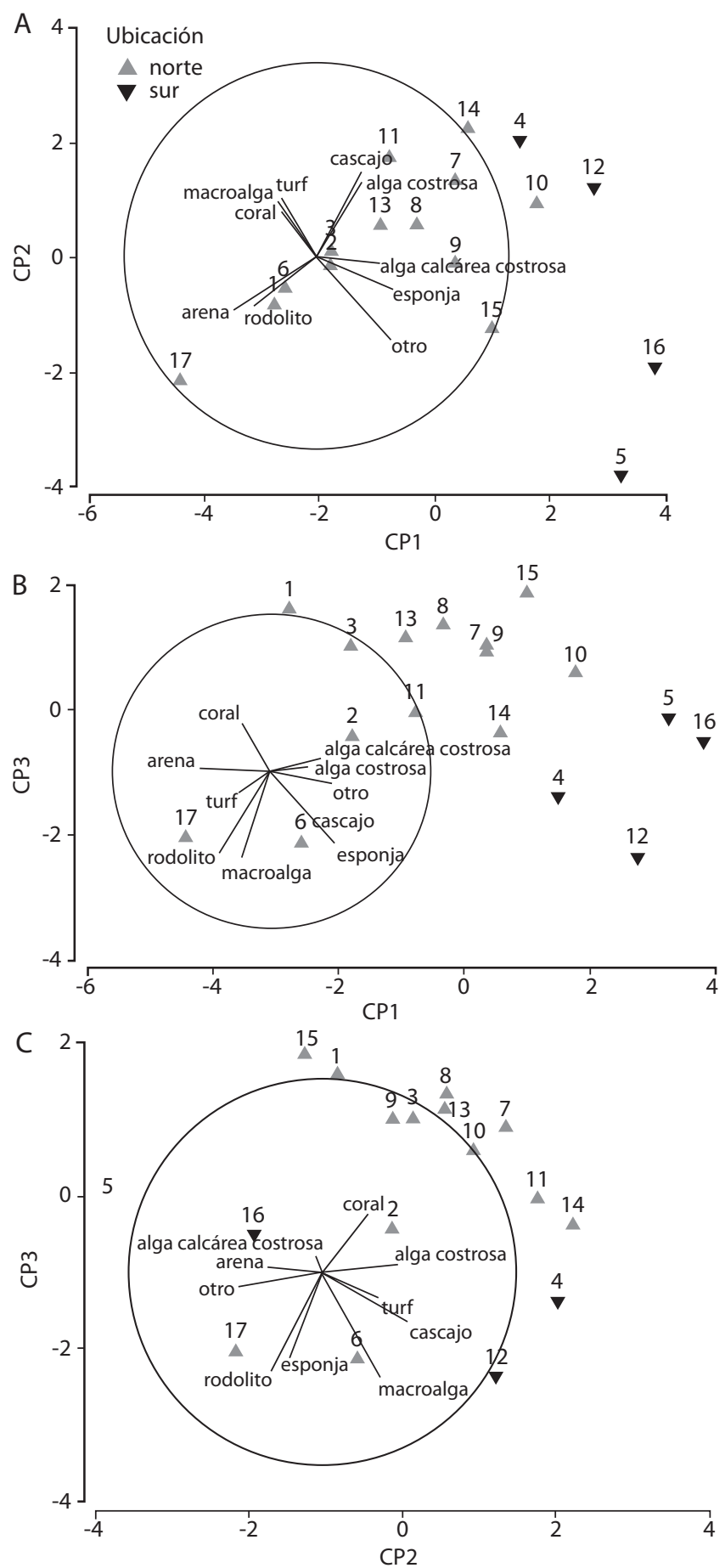

Fig. 3. Análisis de componentes principales (CP) basado en los porcentajes de cobertura del sustrato, utilizando como factor ubicación. (A) CP 1 vs CP 2 (B) CP 1 vs CP3 (C) CP2 vs CP3. Número de los sitios en el Cuadro 1.

Fig. 3. Principal component (PC) analysis based on substrate cover using locality as a factor. (A) PC 1 vs PC 2 (B) PC1 vs PC3 (C) PC22 vs PC3. Site numbers in Table 1. 


\section{CUADRO 4}

Análisis de variancias de una vía en rangos (prueba de Wilcoxon) entre los sitios de muestreo de acuerdo a la ubicación y exposición en el PNIC

TABLE 4

Wilcoxon One Way Analysis of Variance Rank based on location and exposition between sampled sitesat PNIC

\begin{tabular}{cccccc} 
& \multicolumn{2}{c}{ Ubicación } & \multicolumn{2}{c}{ Exposición } \\
$\mathrm{S}$ & Norte & & Sur & $\mathrm{Si}$ & No \\
$\chi^{2}$ & $7.15 \pm 3.26$ & & $5.25 \pm 1.50$ & $5.22 \pm 2.68$ & $8.38 \pm 2.56$ \\
$\mathrm{P}$ & & 1.88 & & 4.57 & \\
$\mathrm{H}^{\prime}\left(\log _{10}\right)$ & & 0.17 & & 0.03 & \\
$\chi^{2}$ & & & $0.24 \pm 0.14$ & $0.37 \pm 0.24$ & $0.58 \pm 0.21$ \\
$\mathrm{P}$ & & 5.31 & & 2.48 & \\
$\Delta^{*}$ & & 0.02 & & 0.12 & $87.73 \pm 2.48$ \\
$\chi^{2}$ & $86.72 \pm 6.88$ & & $86.00 \pm 13.81$ & $85.35 \pm 12.13$ & \\
$\mathrm{P}$ & & 0.06 & & 0.1 & \\
\hline
\end{tabular}

*Todas las pruebas se realizaron con un grado de liberad.

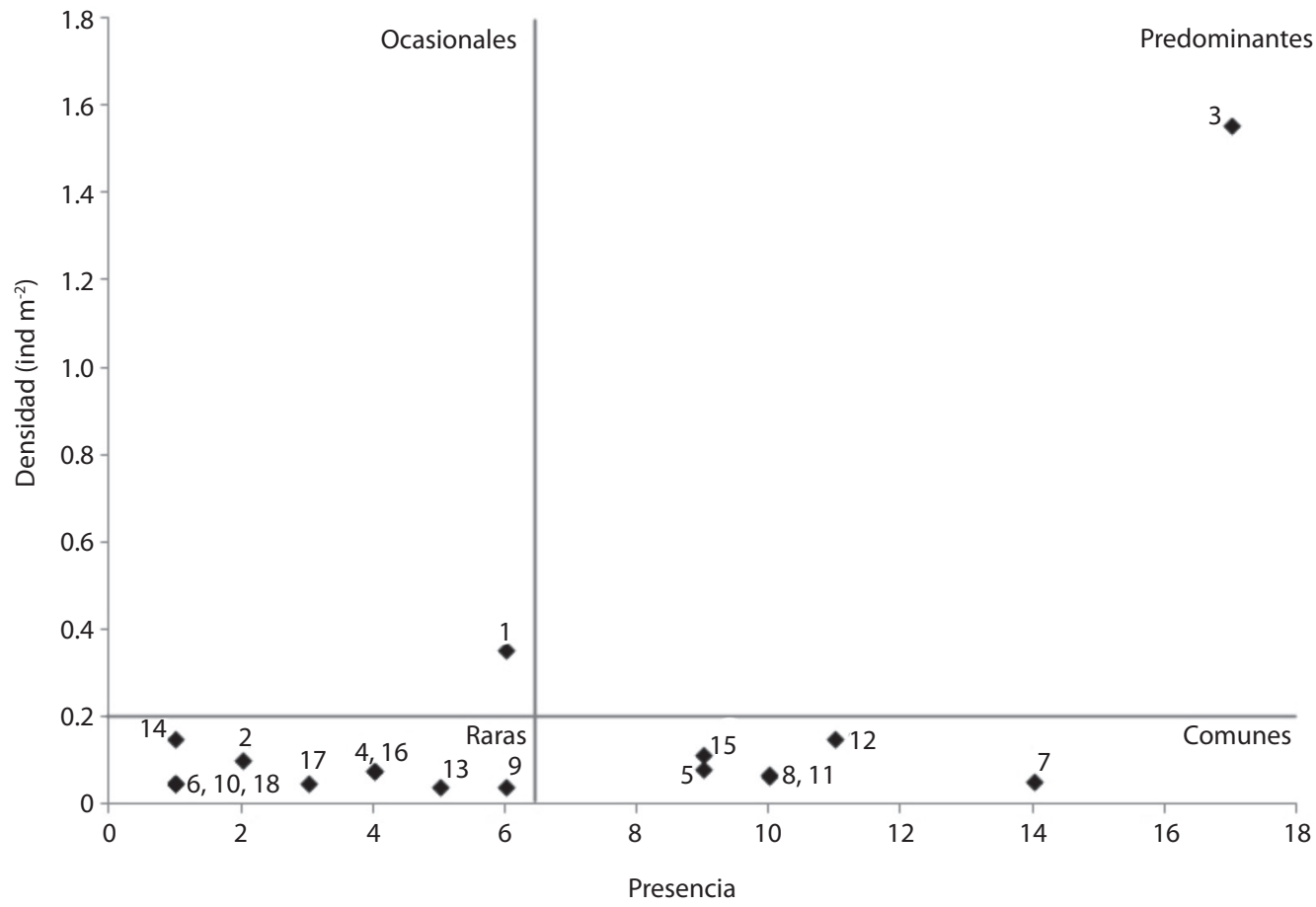

Fig. 4. Categorías de abundancia de macroinvertebrados en el PNIC, basadas en la densidad (ind $\mathrm{m}^{-2}$ ) y la presencia (número de sitios donde la especie fue observada) de todas las especies. Líneas grises indican el valor promedio de la densidad y de la presencia. Número de las especies en el Anexo 2.

Fig. 4. Macroinvertebrate abundance categories at PNIC, based on densities (ind $\mathrm{m}^{-2}$ ) and occurrence (number of sites where the species was observed). Gray lines show mean values of densities and occurrence. Species numbers in Appendix 2. 
embargo los sitios de muestreo no expuestos presentaron mayor riqueza de especies promedio $(8.38 \pm 2.56)$ que los expuestos $(5.22 \pm 2.68)$ (Cuadro 4).

Bahía Chatham, Punta Gissler, Isla Manuelita Adentro, Punta Presidio y Bahía Wafer fueron los sitios que presentaron el valor más alto del índice de diversidad de Shannon (H'). Los sitios al norte de la Isla del Coco presentaron mayor diversidad de especies (Cuadro 4), sin embargo, no se encontraron diferencias significativas en el promedio por sitio respecto a la exposición (Cuadro 4).

Los valores más altos de índice de distintividad taxonómica $\left(\Delta^{*}\right)$ se observaron en Roca Sumergida, Punta Leonel, Punta María, Isla Ulloa y Punta Gissler. No se encontraron diferencias significativas en el $\Delta^{*}$ promedio por sitio respecto a la ubicación o exposición (Cuadro 4).

La mayor similitud de especies (89\%) se encontró entre los sitios Dos Amigos Pequeño, Isla Manuelita Afuera y Roca Sucia, estos a su vez se agruparon junto a Roca Sumergida, Isla Ulloa y Bahía Yglesias (Fig. 5). Una segunda agrupación estuvo compuesta por Bahía Weston, Isla Pájara, Punta Leonel, Punta María, Isla Vikinga, Bahía Wafer, Punta Presidio y Rodolitos. Por último, Bahía Chatham, Punta
Gissler e Isla Manuelita Adentro fueron los sitios con menor similitud entre ellos y con los otros grupos o sitios (Fig. 5). La ubicación de los sitios no afectó la composición de macroinvertebrados $(\mathrm{R}=-0.07, \mathrm{p}=0.63)$, y la composición de macroinvertebrados en los sitios difiere de manera leve con respecto a la exposición $(\mathrm{R}=0.17, \mathrm{p}<0.05)$. De acuerdo al SIMPER, los taxones que más contribuyeron a la similitud entre los sitios expuestos y no expuestos fueron: D. mexicanum, L. columbiae, Hyotissa hyotis, Tripneustes depressus, Spondylus sp., Eucidaris thouarsii y Holothuria (Halodeima) atra (Cuadro 5).

Diversidad y biomasa de peces arrecifales: Se observaron un total de 45338 individuos de peces en los ecosistemas coralinos de la Isla del Coco, distribuidos en 93 especies de 37 familias (Anexo 3). La riqueza de especies promedio por transecto fue de $15.97 \pm 4.01$. Se encontró un promedio de biomasa de $28.32 \pm 151.16$ tn transecto $^{-1}$.

Un $51 \%$ de las especies fueron poco frecuentes y presentaron poca abundancia por lo que se categorizaron como raras (Fig. 6). Un $8 \%$ de las especies fueron ocasionales y $31 \%$ fueron comunes. Por su parte, solo diez especies (11\%) fueron predominantes (Fig. 6).

\section{CUADRO 5}

Análisis de similitud porcentual utilizando densidad de macroivertebrados (ind $\mathrm{m}^{-2}$ ) por el factor exposición en el PNIC

TABLE 5

Analysis of Similarities of macroinvertebrates densities (ind $\mathrm{m}^{-2}$ ) by exposition factor at PNIC

\begin{tabular}{|c|c|c|c|}
\hline Exposición & $\%$ Similitud & Taxones & \% Contribución \\
\hline Sí & 60.65 & Diadema mexicanum & 89.90 \\
\hline \multirow[t]{4}{*}{ No } & 61.69 & Diadema mexicanum & 65.29 \\
\hline & & Linckia columbiae & 8.50 \\
\hline & & Hyotis sahyotis & 5.49 \\
\hline & & Spondylus sp. & 5.23 \\
\hline Exposición & $\%$ Disimilitud & Taxones & \% Contribución \\
\hline \multirow[t]{7}{*}{ Si-No } & 42.39 & Diadema mexicanum & 33.94 \\
\hline & & Linckia columbiae & 15.00 \\
\hline & & Hyotis sahyotis & 7.25 \\
\hline & & Tripneustes depressus & 6.83 \\
\hline & & Spondylus sp. & 6.25 \\
\hline & & Eucidaris thouarsii & 5.90 \\
\hline & & Holothuria (Halodeima) atra & 5.62 \\
\hline
\end{tabular}




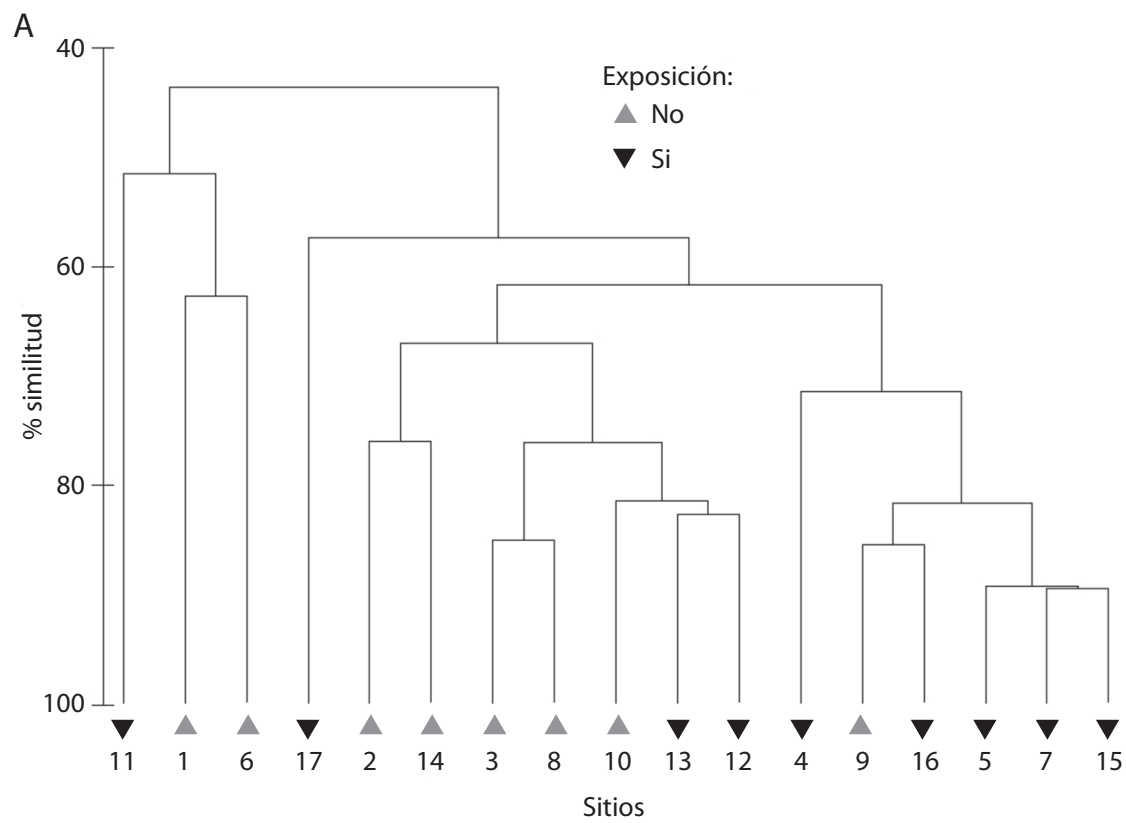

B Nivel de estrés $=0.09$

$\%$ de similitud

70

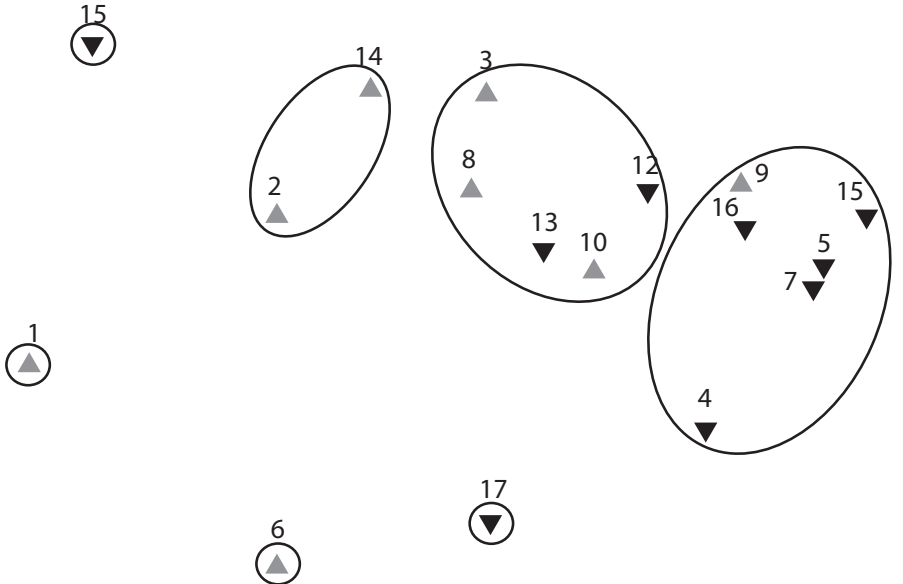

Fig. 5. (A) Dendrograma de unión simple (B) análisis multidimensional escalado por factor exposición, basados en una matriz de similitud de Bray-Curtis utilizando los valores de densidad de macroinvertebrados. Número de los sitios en el Cuadro 1.

Fig. 5. (A) Hierarchical cluster analyses (B) Non metric multi-dimentional scaling analysis by exposition factor, based on a Bray Curtis similarity matrix using macroinvertebrate densities values. Site numbers in Table 1.

Una de esas especies predominantes fue el pez coralívoro Arothron meleagris, mismo que se observó en todos los sitios, con un máximo de 11 individuos en un transecto y un promedio de densidad de 529.20 \pm 407.01 ind $\mathrm{ha}^{-1}$.
Al ordenar los sitios de la Isla del Coco basado en la biomasa por comunidad de peces, la mayoría de los sitios más protegidos se agruparon, contrario a los sitios expuestos más dispersos en cuanto a composición (Fig. 7). Sin 


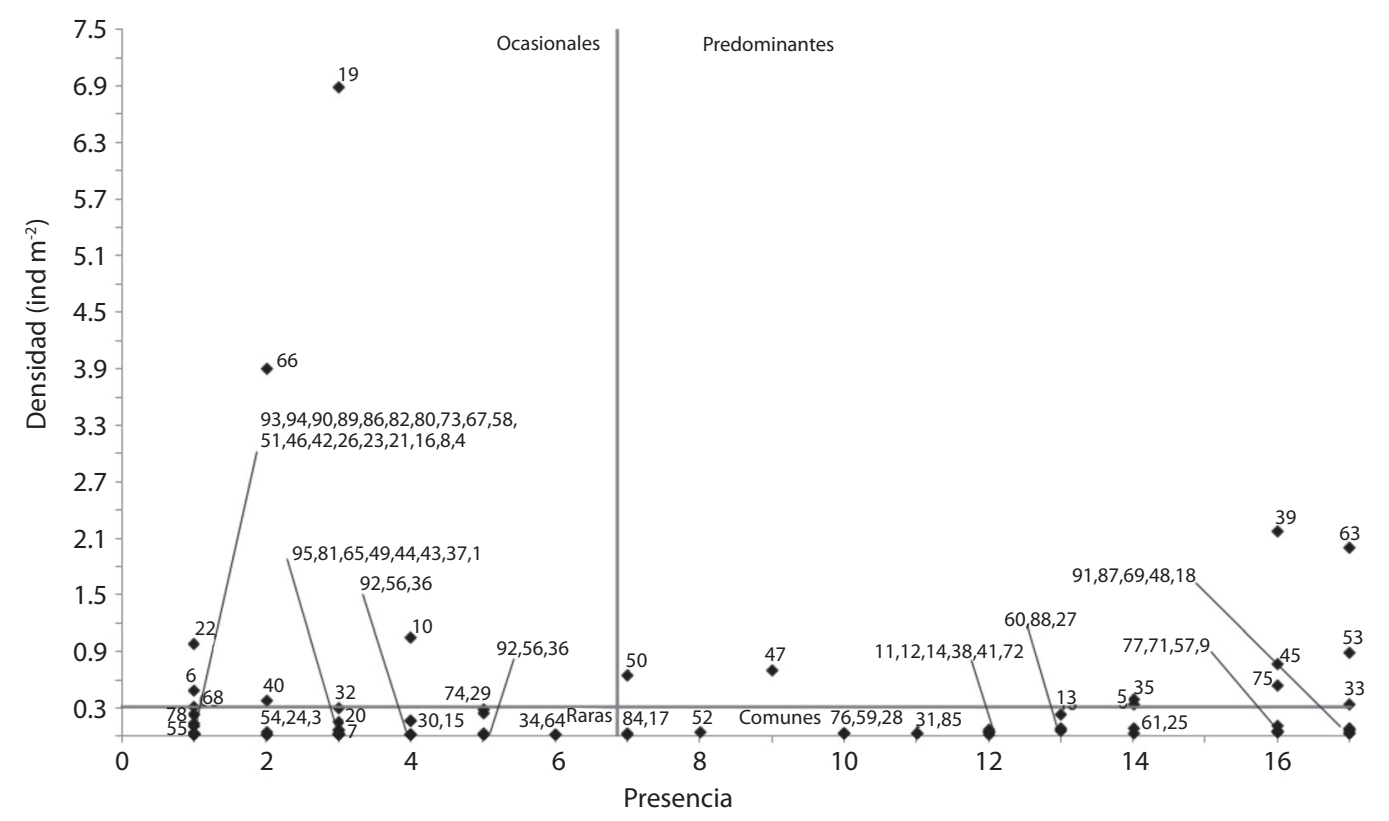

Fig. 6. Categorías de abundancia de peces arrecifales en el PNIC, basadas en la densidad (ind $\mathrm{m}^{-2}$ ) y la presencia (número de sitios donde la especie fue observada) de todas las especies. Líneas grises indican el valor promedio de la densidad y de la presencia. Número de las especies en el Anexo 3.

Fig. 6. Reef fish abundance categories at PNIC, based on densities (ind $\mathrm{m}^{-2}$ ) and occurrence (number of sites where the species was observed). Gray lines show mean values of density and occurrence. Species numbers in Appendix 3.

embargo, se observó un traslape entre los sitios con diferente grado de exposición, no observándose diferencias entre ambos (ANOSIM, $\mathrm{R}=0.114, \mathrm{p}=0.06$ ).

La comunidad de peces del PNIC durante el periodo de estudio estuvo representada por una gran cantidad de especies carnívoras, seguido por depredadores tope, herbívoros y planctívoros, estos últimos con solo siete especies (Cuadro 6). En cuanto al número de individuos muestreados, los carnívoros y depredadores tope mostraron la mayor y menor cantidad respectivamente (Cuadro 6). En contraste, las biomasas fueron dominadas por depredadores tope con más de 2 tn ha ${ }^{-1}$, seguido de planctívoros y herbívoros que presentaron biomasas intermedias. Los carnívoros presentaron los valores menores de biomasa. Se observaron diferencias en cuanto a la biomasa de los grupos tróficos $(\mathrm{F}=2.96$, g.l. $=652$, $\mathrm{p}=0.03$, Fig. 8).

De acuerdo a las categorías de la Lista Roja de la UICN de especies amenazadas de peces, en el PNIC, $16 \%$ especies se encuentran en las categorías amenazadas, que agrupa a casi amenazadas (NT), vulnerables (VU) o amenazadas (EN) (Cuadro 7). Estas especies corresponden principalmente a tiburones, rayas y pequeños peces endémicos de la isla. Estas especies representan un $25 \%$ de la biomasa total muestreada (Cuadro 7). En los censos se observaron varias especies de tiburones (Anexo 3) como el martillo (Sphyrna lewini), tigre (Galeocerdo cuvier), ballena (Rhincodon typus) y punta blanca de arrecife (Trianodon obesus), todos ellos en alguna categoría de amenaza de la IUCN. Otras especies de tiburones como el galápagos (Carcharhinus galapagensis), piloto (Carcharhinus falciformis), punta blanca oceánico (Carcharhinus albimarginatus) y punta negra (Carcharhinus limbatus) también fueron observados en los sitios de muestreo, sin embargo no fueron censadas en los transectos y consecuentemente no fueron cuantificados, por lo cual tanto el número de especies como las biomasas de especies amenazadas (EN, 

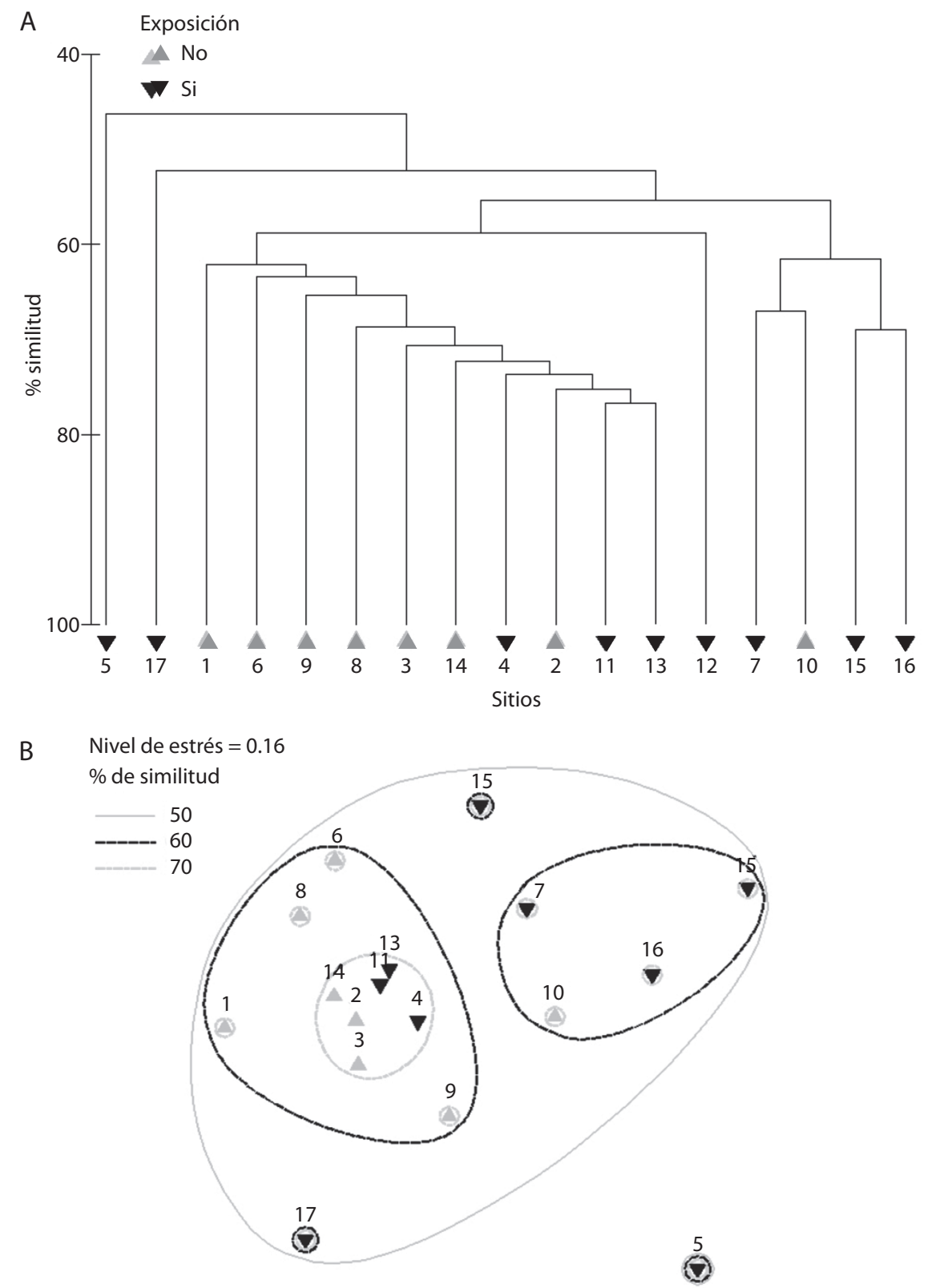

Fig. 7. (A) Dendrograma de unión simple (B) análisis multidimensional escalado por factor de exposición, basados en una matriz de similitud de Bray-Curtis utilizando los valores de biomasa de peces (transformados los datos con raíz cuadrada). Número de los sitios en el Cuadro 1.

Fig. 7. (A) Hierarchical cluster analyses (B) Non metric multi-dimensional scaling analysis by exposition factor, based on a Bray Curtis similarity matrix using fish biomass values (data square root transformed). Site numbers in Table 1. 
CUADRO 6

Número de especies de peces y total de individuos por grupo trófico en el PNIC

TABLE 6

Number of fish species and total individuals per trophic group at PNIC

\begin{tabular}{lcccc}
\multicolumn{1}{c}{ Grupo trófico } & $\begin{array}{c}\text { Total especies } \\
\text { en el grupo }\end{array}$ & $\begin{array}{c}\text { \% del total } \\
\text { de especies }\end{array}$ & $\begin{array}{c}\text { Total individuos } \\
\text { observados }\end{array}$ & $\begin{array}{c}\text { Individuos } \\
\text { observados }(\%)\end{array}$ \\
Carnívoro & 36 & 38 & 15339 & 34 \\
Depredador Tope & 29 & 31 & 7074 & 16 \\
Herbívoro & 22 & 23 & 10997 & 24 \\
Planctívoro & 7 & 7 & 11927 & 26 \\
\hline
\end{tabular}

\section{CUADRO 7}

Número de especies de peces, biomasa total y promedio (desviación estándar) de la biomasa por transecto en el PNIC de acuerdo a las categorías de la Lista Roja de la UICN

TABLE 7

Number of fish species, total biomass and average (standard deviation) biomass per transect at PNIC according to the IUCN Red List

\begin{tabular}{cccc} 
Categoría UICN & Número de especies & Biomasa total $\left(\mathrm{tn} \mathrm{ha}{ }^{-1}\right)$ & Biomasa promedio $\left(\mathrm{tn} \mathrm{ha}^{-1}\right)$ \\
EN & 1 & 288.71 & $36.09 \pm 16.14$ \\
LC & 53 & 1585.64 & $9.33 \pm 26.53$ \\
NE & 26 & 591.97 & $3.5 \pm 4.89$ \\
NT & 7 & 411.97 & $5.02 \pm 6.15$ \\
VU & 8 & 16.54 & $0.23 \pm 1.73$ \\
\hline
\end{tabular}

$\mathrm{DD}=$ Datos insuficientes; $\mathrm{NE}=\mathrm{No}$ evaluado; NT=Casi amenazado; LC=Preocupación menor; VU=Vulnerable; EN=En peligro.

$\mathrm{DD}=$ Data deficient; NE=Not evaluated; NT=Near threatened; LC=Least concern; VU=Vulnerable; EN=Endangered.

NT, VU) debe ser aún mayor que lo reportado en este trabajo.

\section{DISCUSIÓN}

El valor biológico que posee el Parque Nacional Isla del Coco es reconocido a nivel mundial (Edgar et al., 2014). Esto posiblemente se debe a que esta área protegida esté funcionando contra tres tipos de extinción, la local, la ecológica y la comercial (McCauley et al., 2015). Dentro de sus aguas, observamos arrecifes saludables, con cadenas tróficas completas y funcionales; donde las especies son abundantes lo que les permite que cumplan sus papeles funcionales, y donde especies de alto valor comercial se encuentran en buen estado poblacional.
Cobertura del fondo y rugosidad: En la Isla del Coco, actualmente, la cobertura promedio de coral vivo ronda $18.64 \%$ (ámbito 2.89-55.44). En la Isla de Coiba y en el Archipiélago de las Perlas, Panamá, la cobertura de coral vivo reportada es de $37.3 \%$ (1.2-76.1 $\%)$ y $61.2 \%(0.1-96.4 \%)$ respectivamente (Guzman, Guevara, \& Breedy, 2004; Guzman, Benfield, Breedy, \& Mair, 2008a), siendo superiores a lo observado en el PNIC. Sin embargo, la Isla del Coiba y sus islas e islotes asociados miden $535.82 \mathrm{~km}^{2}$ de territorio insular (Guzman et al., 2004), y el Archipiélago de las Perlas $1165 \mathrm{~km}^{2}$ (Guzman et al., 2008a), mientras que la Isla del Coco mide $23.85 \mathrm{~km}^{2}$ (Guzman, \& Cortés, 1992).

El comportamiento temporal de la cobertura de coral vivo en Isla del Coco evidencia su 


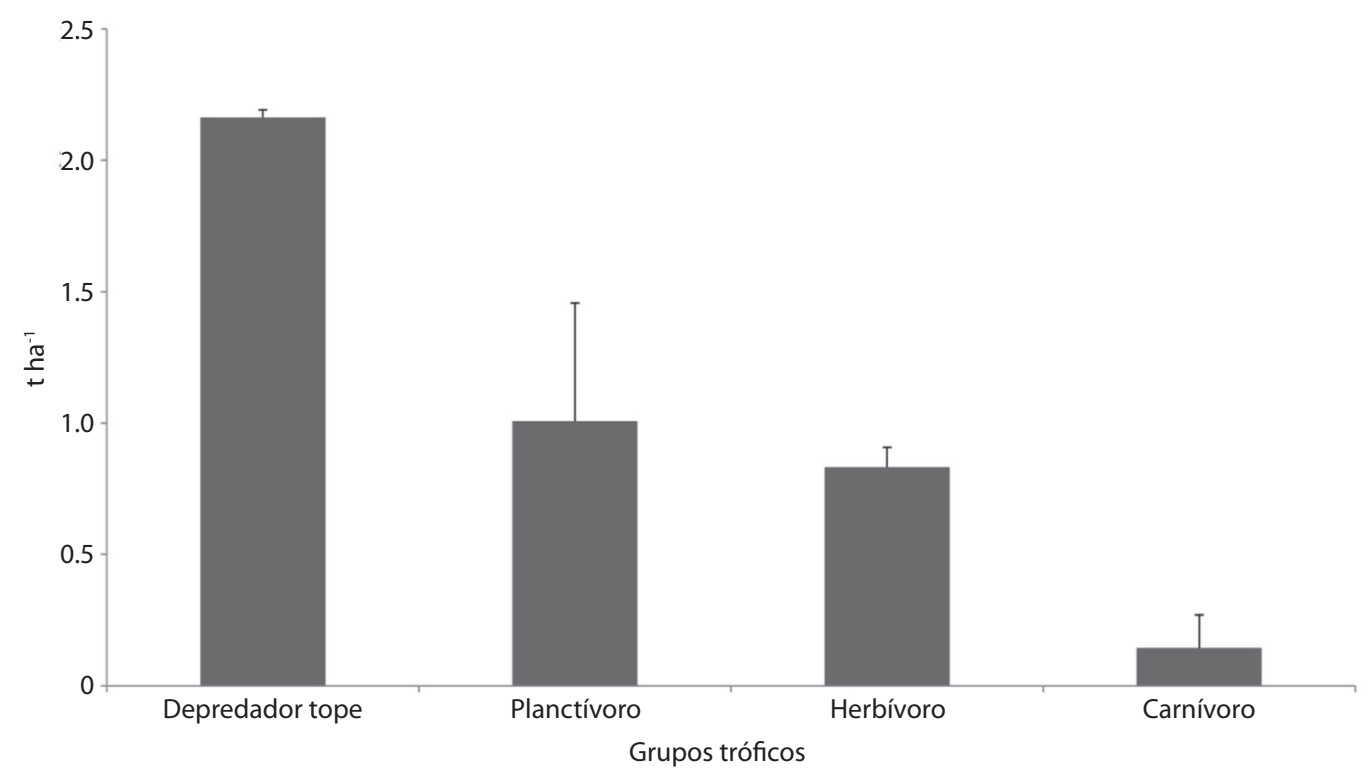

Fig. 8. Promedio ( \pm error estándar) de la biomasa de los grupos tróficos de peces en el PNIC.

Fig. 8. Mean biomass ( \pm standard error) of fish trophic groups at PNIC.

recuperación. El Niño de 1987-1988, produjo una alta mortalidad coralina dejando vivo solamente $4 \%$ de coral y la cobertura de TA alcanzó un $89 \%$ (Guzman, \& Cortés, 1992) (Cuadro 8). Para el 2002, la CCV mostró un incremento alcanzando $22 \%$, el TA disminuyó a $64 \%$ y la cobertura de ACC fue de $5.7 \%$ (Guzman, \&
Cortés, 2007) (Cuadro 8). Estas evaluaciones de 1987 y 2002 se realizaron en cinco sitios de la isla: Punta Presidio, Bahía Chatham, Punta Pacheco, Isla Pájara y Punta Gissler (Guzman, \& Cortés, 1992; 2007). Estos mismos sitios fueron evaluados en la presente investigación, con la excepción de Punta Pacheco, y

\section{CUADRO 8}

Comparación del porcentaje (\%) promedio (desviación estándar) de la cobertura de coral vivo a través del tiempo en cinco sitios del PNIC, y comparación del porcentaje (\%) promedio (desviación estándar) de la cobertura de cuatro categorías de sustrato

TABLE 8

Comparison of average (standard deviation) percentage (\%) of live coral cover over time at five sites at PNIC, and comparison of the average (standard deviation) percentage (\%) of four substrate categories

\begin{tabular}{lccc} 
& $1987^{1}$ & $2002^{2}$ & $2013-2014^{3}$ \\
Punta Presidio & $3.47 \pm 0.27, \mathrm{n}=72$ & $25.70 \pm 1.00, \mathrm{n}=5$ & $18.64 \pm 14.65, \mathrm{n}=15$ \\
Bahía Chatham & $2.91 \pm 0.42, \mathrm{n}=80$ & $16.70 \pm 0.87, \mathrm{n}=5$ & $34.27 \pm 9.16, \mathrm{n}=6$ \\
Punta Pacheco & $2.60 \pm 0.35, \mathrm{n}=147$ & $2.36 \pm 0.27, \mathrm{n}=5$ & - \\
Isla Pájara & - & $58.70 \pm 2.61, \mathrm{n}=3$ & $55.44 \pm 7.03, \mathrm{n}=15$ \\
Punta Gissler & - & $10.00 \pm 0.96, \mathrm{n}=3$ & $31.87 \pm 4.81, \mathrm{n}=15$ \\
Coral & $2.99 \pm 0.44$ & $22.70 \pm 21.90$ & $36.60 \pm 13.19$ \\
Turf & $89.9 \pm 1.83$ & $64.70 \pm 9.10$ & $38.45 \pm 17.81$ \\
Algas calcáreas costrosas & - & $5.70 \pm 1.20$ & $15.27 \pm 12.11$ \\
Arena & $7.06 \pm 1.96$ & $6.90 \pm 4.30$ & $8.41 \pm 14.57$ \\
\hline
\end{tabular}

1: Guzman \& Cortés 1992; 2: Guzman \& Cortés 2007; 3: Esta investigación. 
presentaron en promedio coberturas de coral vivo de $36 \%$, de TA de $38 \%$ y de ACC de $15 \%$ (Cuadro 8). Indicando una recuperación a través del tiempo en coral vivo y ACC, y una disminución de TA.

Dos argumentos pueden explicar esta recuperación coralina. El primero, que mencionan Guzman \& Cortés (2002) y Alvarado et al. (2012), es la disminución de la presión de la acción bioerosiva de los erizos de mar $D$. mexicanum. En este sentido, los erizos de mar han favorecido el incremento en la cobertura de algas calcáreas, lo que posiblemente brindó el sustrato adecuado para el asentamiento de reclutas de coral (Alvarado et al., 2012). El segundo argumento, y que ya ha sido mencionado anteriormente en el texto, son las medidas de conservación que se han impuesto en la isla (i.e. prohibición total de extracción pesquera), el tamaño del área protegida $(22.2 \mathrm{~km}$ alrededor de la isla) y la distancia de la costa $(>500 \mathrm{~km})$. Este argumento está muy ligado al primero, en el sentido que al no haber otras fuentes de presión sobre estos ambientes, como la sobrepesca o efectos de la población humana costera, las redes tróficas se mantuvieron saludables, lo que a su vez permitió controlar las poblaciones de erizos de mar y que su impacto bioerosivo no fuera mayor. Por lo tanto, el ecosistema arrecifal de la isla tuvo un proceso de recuperación en 20 años, permitiéndole alcanzar valores de cobertura coralina considerados saludables.

\section{Diversidad y abundancia de macroin-} vertebrados: A pesar que la historia de la investigación científica en Isla del Coco se remonta a finales del siglo XIX, con la visita del buque Albatross en 1888 (Cortés, 2008a), es poca la información que se tiene respecto a la dinámica de las comunidades de macroinvertebrados en los arrecifes. En 2008, Cortés resaltó que los aportes científicos en este grupo de organismos han sido principalmente taxonómicos, con la excepción de los trabajos dirigidos al grupo de los corales (Guzman, \& Cortés, 1992, 2007) y los equinodermos (Alvarado, \& Chiriboga, 2008; Alvarado et al., 2012).
Las comunidades de macroinvertebrados del PNIC se pueden dividir respecto al grado exposición de la costa y a la heterogeneidad en la composición de especies. Con base en estas categorías se distinguen tres grupos: 1) sitios expuestos con baja heterogeneidad, 2) sitios no expuestos con heterogeneidad media, y 3 ) sitios no expuestos con alta heterogeneidad. Los sitios expuestos están influenciados por efectos erosivos del oleaje y corrientes más fuertes que los sitios no expuestos, lo que probablemente provoca que disminuya la cantidad de materia orgánica y aumenté la cantidad de carbonatos (Sibaja-Cordero, Troncoso, Benavides-Varela, \& Cortés, 2012).

La ubicación geográfica no resultó ser un factor determinante de la riqueza de especies (S) o de la distintividad taxonómica $\left(\Delta^{*}\right)$, lo que podría ser resultado de los pocos sitios muestreados en la zona sur, o debido a que la isla es bastante homogénea en su composición por su pequeño tamaño. Los sitios ubicados al norte de la isla, principalmente los que se encuentran en las cercanías de las tres bahías (Chatham, Weston y Wafer), presentaron los índices de diversidad más altos. Esta zona se caracteriza por poseer alrededor de los arrecifes, fondos suaves compuestos de arena y lodo con gran cantidad de materia orgánica, proveniente del aporte de los ríos y del detritus del coral (Sibaja-Cordero et al., 2012). Es posible que la acumulación de estos materiales favorezca a las poblaciones de organismos que viven entre los sedimentos, y estos a su vez, permitan la incorporación de más organismos en las redes tróficas de la zona.

De acuerdo con Vargas-Castillo \& Wehrtmann (2008), Isla del Coco cuenta con al menos 135 especies de crustáceos decápodos y estomatópodos. En el presente estudio solo cuantificamos el género de langosta Panulirus, esto debido principalmente al método empleado para las observaciones. Actualmente se tienen registradas tres especies de langostas para la Isla del Coco, un poliquélido y dos palinúridos, Panuliris gracilis y Panulirus penicillatus (Vargas-Castillo, \& Wehrtmann, 2009). La langosta es un recurso de alto valor comercial 
que experimenta una gran extracción a lo largo del PTO (Guzman, Cipriani, Vega, López, \& Mair, 2008b). Esta alta extracción ha provocado que en los arrecifes continentales sea un recurso escaso. En Coiba y Las Perlas, Panamá, Guzman et al. (2008b) informan densidades de 7.6 y 4.1 ind ha $^{-1}$ respectivamente, las cuales son consideradas bajas versus otras zonas del PTO. En Guerrero, México, Briones-Fourzán \& Lozano-Álvarez (2000) reportan densidades entre 6.6 y 43.1 ind ha ${ }^{-1}$, mientras en las Islas Galápagos las densidades rondan entre 1 y 55 ind ha $^{-1}$ (Edgar, Banks, Fariña, Calvopiña, \& Martínez, 2004). Estos ámbitos de sitios explotados con densidades bajas $\left(<10\right.$ ind $\left.\mathrm{ha}^{-1}\right)$, y sitios con densidades altas ( $>40$ ind ha $\left.{ }^{-1}\right)$, son más evidentes al comparar zonas arrecifales continentales versus oceánicas, donde en los últimos sitios las densidades pueden alcanzar en promedio aproximadamente los 350 ind $\mathrm{ha}^{-1}$, mientras que los arrecifes continentales no llegan a superar los 50 ind ha-1 (Edgar et al., 2011). Esto evidencia cierta presión pesquera sobre las poblaciones de langosta en los arrecifes continentales, mientras que en las islas oceánicas como (Coco, Malpelo y ciertas zonas de Galápagos), la presión pesquera es menor y por lo tanto las poblaciones tienden a ser mayores. En nuestra investigación la densidad promedio de langosta fue de $388 \pm 385$ ind ha ${ }^{-1}$, siendo la más alta de la región.

Este mismo aspecto se puede observar con la densidad de pepinos de mar, específicamente con la especie Isostichopus fuscus, la cual posee un alto valor comercial (Purcell, Polidoro, Hamel, Gamboa, \& Mercier, 2014), y en la isla posee una densidad promedio de 550 ind ha $^{-1}$. Esta densidad es menor a la observada en las Islas Galápagos, 833 ind ha-1 ${ }^{-1}$ Edgar et al., 2004), y a su vez es superior a la determinada en el Parque Nacional Bahía de Loreto (280 ind ha ${ }^{-1}$ ), en Baja California Sur, México (Reyes-Bonilla, Herrero-Pérezrul, GonzálezRomero, González-Peralta, \& Ramírez-Hernández, 2008). Estas densidades refuerzan el hecho de que el PNIC está funcionando como un reservorio de especies de interés comercial cuyas poblaciones en otras zonas arrecifales de la región se encuentran diezmadas.

El erizo de mar $D$. mexicanum fue el macroinvertebrado predominante y que presentó las mayores densidades en la isla. Su presencia ha sido clave en la recuperación de los arrecifes coralinos de este lugar. Alvarado et al. (2012) presenta como las densidades de este organismo han variado a través del tiempo, y se han mantenido estables a la fecha. Es evidente que las densidades actuales (1.77 ind $\mathrm{m}^{-2}$ ), son notablemente inferiores a las observadas en 1987 (14.5 ind $\mathrm{m}^{-2}$ ) (Guzman, \& Cortés, 1992; 2007). Esta disminución en la poblaciones de $D$. mexicanum puede ser, como se mencionó anteriormente, el producto de una buena salud arrecifal donde existe presencia de peces depredadores del erizo (i.e. A. meleagris, Diodon holocanthus, Pseudobalistes naufragium, Sufflamen verres, entre otros) que logran controlar las poblaciones.

Otro equinodermo, con un valor ecológico importante, es la estrella coralívora Acanthaster planci, que durante las evaluaciones de esta investigación no se observaron en los transectos, aunque si fueron percibidas algunas fuera de ellos. Guzman \& Cortés (1992) observaron a esta estrella alimentándose de $P$. lobata en 1987 con una densidad promedio de 10.4ind $\mathrm{m}^{-2}$. En el 2002, Guzman \& Cortés (2007) reportan una densidad promedio de 7.4ind $\mathrm{m}^{-2}$, mientras que Alvarado \& Chiriboga (2008) indican una densidad de 0.02 ind $\mathrm{m}^{-2}$. Estudios realizados en Australia con A. planci advierten un decaimiento en las poblaciones debido al calentamiento de global, porque la muerte de coral limita la alimentación de la misma, y temperaturas por encima de $28.5^{\circ} \mathrm{C}$ afectan significativamente el desarrollo su larval (Lemare et al., 2014). Sin embargo, la cobertura coralina en la isla es alta y predominan especies de coral que son presas preferidas de la estrella (Glynn, 1974), por lo tanto el factor que puede estar afectando a la estrella son altas temperaturas que estén provocando algún fallo en su desarrollo larval. Sin embargo, este punto debe comprobarse con mayor investigación. 
Diversidad y biomasa de peces arrecifales: La lejanía de la costa y los esfuerzos por regular la pesca ilegal en sus aguas ha permitido que el PNIC sea uno de los sitios con mayores biomasas de peces que existe (Friedlander et al., 2012). Friedlander et al. (2012) visitaron el PNIC en setiembre del 2009, y evaluaron 29 sitios donde realizaron transectos de banda de $25 \mathrm{~m}$ a dos profundidades y encuentran 87 especies de peces arrecifales con una biomasa promedio de 7.6 \pm 7.6 tn ha ${ }^{-1}$. De estos 29 sitios, 11 son islotes o pináculos que se encuentran en aguas abiertas o alejados de la costa, con características más oceánicas, y donde la presencia de peces pelágicos es mayor. Así mismo, el ámbito de profundidad que se cubrió llegó hasta $\operatorname{los} 33 \mathrm{~m}$. En nuestra investigación, para la zona arrecifal con características costeras hasta $17 \mathrm{~m}$ de profundidad, cuantificamos 93 especies con una biomasa promedio de $1.2 \pm 8.5$ tn $^{h^{-1}}$ en 17 sitios, donde la presencia y diversidad de peces pelágicos es menor. Es claro que por la diferencia en ambientes muestreados la biomasa que nosotros determinamos es menor a la observada por Friedlander et al. (2012), pero podemos decir que la biomasa encontrada en nuestra investigación fue alta si se compara con otras zonas arrecifales del PTO (Edgar et al., 2011). En localidades oceánicas con alto nivel de protección, la biomasa promedio de depredadores tope es de $\sim 1.4$ tn ha $^{-1}$ (Edgar et al., 2011), siendo similar a la observada en este estudio ( 2 tn ha $^{-1}$ ) (Fig. 8). Por su parte, la biomasa de este grupo trófico es mucho menor $\left(<\sim 0.5\right.$ tn ha $\left.{ }^{-1}\right)$ en localidades costeras, ya sea que sean protegidas o abiertas a la pesca, o en localidades oceánicas con poca o ninguna protección (Edgar et al., 2011).

Los herbívoros son un grupo trófico de peces que evidencia la salud del ecosistema marino en la isla, en términos comparativos con otras localidades. En nuestra evaluación, la biomasa de este grupo fue en promedio de 1 tn ha- ${ }^{-1}$, siendo muy similar a la reportada por Edgar et al. (2011) ( 0.8 tn ha $\left.^{-1}\right)$ para localidades oceánicas con alto nivel de protección a lo largo del Corredor Marino de Conservación del PTO. Estos resultados indican que un alto grado de protección produce una alta biomasa de peces, y segunda, que es posible que la biomasa total de peces de la isla sea una de las mayores de todo el PTO.

Después de años de extracción pesquera cerca de las zonas costeras de la Isla del Coco, las poblaciones de peces en los arrecifes se han podido recuperar gracias a la declaratoria de Parque Nacional, hasta llegar a un funcionamiento semejante a sitios prístinos (Sandin et al., 2008; Sibaja-Cordero, 2008; Friedlander et al., 2012). Esto se ve reflejado en la estructura trófica de la isla, que muestra una pirámide invertida, con una predominancia de altas biomasas de depredadores tope en los arrecifes que ronda las $1.2 \pm 8.5 \mathrm{tn} \mathrm{ha}^{-1}$, como lo ha demostrado este estudio, y que puede llegar hasta 6 tn ha ${ }^{-1}$ al muestrear sitios más oceánicos (Friedlander et al., 2012). Estas cantidades de tiburones y otros peces grandes son comunes en ecosistemas marinos con poca afectación antropogénica, y estos grupos constituyen presiones selectivas que mantienen los sistemas productivos y cadenas tróficas equilibradas (Friedlander et al., 2012; Espinoza, Cappo, Heupel, Tobin, \& Simpfendorfer, 2014; White, Myers, Mills-Flemming, \& Baum, 2015). Además, se ha determinado que la cobertura de coral y la cercanía con arrecifes también son factores que favorecen la riqueza y abundancia de tiburones, inclusive de especies que no son residentes en sistemas arrecifales, por lo cual la protección de los ecosistemas arrecifales tiene importantes efectos en las comunidades de peces, aún si son especies altamente móviles (Espinoza et al., 2014).

Se ha visto que la estructura del arrecife en gran parte determina la comunidad de peces, y que los arrecifes dominados por corales masivos como $P$. lobata suelen tener ictiofaunas diversas debido a la estructura compleja brindada por el coral (Palacios, \& Zapata, 2014). Es de esperar que la alta cobertura coralina, principalmente en las bahías de la Isla del Coco, albergue una alta diversidad y biomasa de peces. A pesar de esto, las comunidades de peces no muestran una segregación espacial debida a factores como la exposición al oleaje. 
Esta homogeneidad ha sido observada en otros trabajos caracterizando las comunidades de peces de la Isla del Coco (Friedlander et al., 2012). Las variaciones en la iciofauna se dan temporalmente asociadas a anomalías de temperatura superficial u otros factores ambientales (Sibaja-Cordero, 2008).

En cuanto a la predominancia de especies de peces, destaca el tamboril negro ( $A$. meleagris), él cual es un pez coralívoro facultativo cuyo hábito de morder los corales lo hace una especie potencialmente clave en el monitoreo de la salud de los arrecifes del PTO (Guzman, \& Cortés, 1992; 2007; Glynn, Enochs, Afflerbach, Brandtneris, \& Serafy, 2014; Palacios, Muñoz, \& Zapata, 2014). Se ha observado un aumento en la cantidad de $A$. meleagris de $8.2 \pm 0.5$ ind ha ${ }^{-1}$ en 1987 (Guzman, \& Cortés, 1992), $152.6 \pm 37.5$ ind ha $^{-1}$ en 2002 (Guzman, \& Cortés, 2007), y 529.20 \pm 407.01 ind $\mathrm{ha}^{-1}$ en 2013-2014 (presente investigación). Este aumento ha estado acompañado de una recuperación de la cobertura de coral después de los eventos de El Niño, sin que exista una afectación por parte de este crecimiento poblacional sobre el reclutamiento coralino (Guzman, \& Cortés, 2007). Glynn (1997) la cataloga como una especie bioerosionadora, que consume cerca de $20 \mathrm{~g}$ de coral diario, provocando, con densidades bajas, una pérdida de $30 \mathrm{~g}$ de $\mathrm{CaCO}_{3} \mathrm{~m}^{-2}$ al año. Además, se ha determinado que incluso pequeñas abundancias de esta especie pueden afectar la recuperación de los arrecifes debido al consumo de reclutas de coral (Glynn et al., 2014). Sin embargo, también se le reconocen beneficios ya que quiebran los corales y favorece la reproducción y propagación asexual (Guzman, \& Cortés, 2007). Adicionalmente, se ha determinado que su efecto depredador incrementa la variabilidad morfológica de corales del género Pocillopora, favoreciendo un incremento en su cobertura coralina (Palacios et al., 2014). Este género de coral fue abundante en el PNIC previo al impacto de El Niño de 1982-1983, el cual produjo una drástica reducción de su cobertura (Guzman, \& Cortés, 1992; 2007). En la actualidad esta especie de coral muestra signos de recuperación en el Parque. Un fenómeno similar se ha observado en arrecifes coralinos afectados por El Niño en el Pacífico de Panamá (Glynn et al., 2014). Edgar et al. (2011) en su evaluación regional del PTO, indica que esta especie de pez posee mayores densidades en localidades protegidas $\left(44.5 \pm 17.1\right.$ ind ha $\left.^{-1}\right)$ que en localidades con pesca ( $7.63 \pm 6.37$ ind ha $\left.{ }^{-1}\right)$. Es importante investigar más a fondo las relaciones entre las fluctuaciones de la cobertura de coral y las densidades de esta especie para determinar si su presencia en la Isla del Coco puede causar un alto impacto por bioerosión o, por el contrario, su presencia y altas densidades estén más ligadas a favorecer una recuperación coralina y por lo tanto su presencia sea un indicador de buena salud arrecifal.

Los tiburones son la fauna de peces más emblemática de la Isla del Coco debido a su gran diversidad y a las grandes agregaciones de especies, como por ejemplo el tiburón martillo (Sibaja-Cordero, 2008; Zanella, LópezGarro, Golfín-Duarte, \& Saenz, 2012). La mayor parte de los turistas van al PNIC por el buceo con tiburones y las actividades económicas asociadas al turismo en la isla genera cerca de $\$ 8$ millones de ingresos brutos al año (Moreno-Díaz, 2012). En el presente trabajo se observaron varias especies de tiburones, sin embargo solo cuatro fueron cuantificados en los transectos. Una de ellas, el tiburón tigre, hasta hace pocos años empezó a ser más común. En contraste, especies como el tiburón piloto que tradicionalmente ha sido común ya casi no se observa y las densidades son bajas (Sibaja-Cordero, 2008; Friedlander et al., 2012; White et al., 2015).

Otro aspecto importante de recalcar en el PNIC es que alberga una cantidad grande de especies en alguna categoría dentro de la Lista Roja de especies de la Unión Internacional de Conservación de la Naturaleza (UICN, 2001). Esto se debe en gran medida a las especies endémicas de la Isla, las cuales en su mayoría son especies pequeñas de peces sedentarios por lo cual la protección de los arrecifes y lechos de rodolitos que hay en la isla son importantes para mantener esta diversidad (Friedlander 
et al., 2012). Otro componente importante de estas especies sensibles son los tiburones cuyas poblaciones a nivel mundial han sido amenazadas por la pesca, y a pesar de los esfuerzo de conservación, el PNIC no escapa a este problema (Friedlander et al., 2012). En este sentido el gobierno de Costa Rica está tomando acciones para proteger los recursos pesqueros de la Isla, por lo cual ha declarado un Área Marina de Manejo que comprende la Isla del Coco y montes submarinos aledaños, esto con el fin de regular las actividades que se desarrollan en la zona y aumentar la protección alrededor de la Isla.

\section{AGRADECIMIENTOS}

La presente investigación no se hubiera podido realizar sin el apoyo en campo de las siguientes personas: A. Ayala, J. A. Sibaja, C. Sánchez Godínez, C. Sánchez Noguera, V. Bogantes, M. C. Díaz, M. A. Quesada, E. Ochoa, K. García, S. Conejo, C. Salas, J. M. Camargo, G. Bunnin, R. Mosquera, E. Solano, P. Miranda, F. Flores, J. L. Monge, J. Pericas, D. Quesada y C. Cruz. Así mismo, el trabajo se vio fortalecido por el apoyo de Conservación Internacional, Undersea Hunter Group, colegas del CIMAR y funcionarios de PNIC. Un especial agradecimiento a Conservación Internacional por creer en este tipo de investigaciones. Este proyecto contó con el apoyo financiero de Conservación Internacional y del CIMAR. Así mismo, contó con el aval del ACMIC a través de la resolución 065-2013-SINAC, y se encuentra inscrito en FUNDEVI (2739-019) y en Vicerrectoría de Investigación de la Universidad de Costa Rica (808-B3-503). Así mismo, agradecemos los comentarios de dos revisores anónimos que mejoraron este trabajo.

\section{RESUMEN}

La Isla del Coco posee uno de los arrecifes coralinos más diversos y en mejor estado de conservación de Costa Rica. Estos arrecifes han sido ampliamente estudiados desde la década de 1980, sin embrago, estas investigaciones se han enfocado en la cobertura coralina. En esta investigación, se realiza una evaluación de los arrecifes coralinos de la isla, estudiando no solo la cobertura del fondo, sino también la composición de peces e invertebrados, así como la complejidad arrecifal. Se visitaron 17 sitios alrededor de la isla en julio 2013 y febrero-marzo 2014. En cada sitio, se trabajaron tres profundidades (4-8 m, 9-12 m y 13-16 m), donde se muestrearon tres transectos de $10 \mathrm{~m}$ de longitud. Los tapetes de algas, compuestos por una combinación de especies de algas rojas, verdes y pardas, predominaron la cobertura del fondo con $38.18 \pm 5.58 \%$. Las algas calcáreas costrosas (Orden Corallinales) presentaron un $28.12 \pm 5.85 \%$ de cobertura y el coral vivo un $18.64 \pm 3.55 \%$, compuesto principalmente por el coral Porites lobata. Se observaron 18 taxones de macroinvertebrados en los sitios estudiados. Un $56 \%$ de las especies fueron poco abundantes y $33 \%$ fueron comunes. El erizo de mar Diadema mexicanum fue la única especie predominante. Destaca la densidad de las langostas del género Panulirus con $388 \pm 385$ ind ha $\mathrm{a}^{-1}$, siendo la más alta del Pacífico Tropical Oriental, así como del pepino de mar Isostichopus fuscus (550 ind $\mathrm{ha}^{-1}$ ), la cual posee un alto valor comercial. Se observaron un total de 45338 individuos de peces, distribuidos en 94 especies y con un promedio de biomasa por transecto de $1.2 \pm 8.5 \mathrm{tn} \mathrm{ha}^{-1}$. Del total de especies, $51 \%$ fueron raras, $31 \%$ comunes, 11 $\%$ predominantes y $8 \%$ ocasionales. La comunidad de peces del Parque Nacional Isla del Coco (PNIC) estuvo representada por una gran cantidad de especies carnívoras, seguido por depredadores tope, herbívoros y planctívoros. De acuerdo a las categorías de la Lista Roja de la UICN de especies amenazadas de peces, $16 \%$ de las especies se encuentran en las categorías amenazadas y representan un $25 \%$ de la biomasa total muestreada. Al comparar los trabajos realizados desde 1987 hasta el presente es evidente la recuperación en la cobertura de coral vivo a través del tiempo, principalmente por: 1) una disminución de la presión de la acción bioerosiva de los erizos de mar D. mexicanum; y 2) las medidas de conservación que se han impuesto en la isla. Esto ha favorecido que los arrecifes sean complejos y rugosos. En general los arrecifes del PNIC son saludables, con cadenas tróficas complejas y funcionales; donde especies de alto valor comercial se encuentran en buen estado de conservación. La efectividad de las actividades de conservación y manejo en la Isla del Coco se pueden visualizar en la recuperación y complejidad de la comunidad arrecifal.

Palabras clave: cobertura de coral vivo, Diadema mexicanum, recuperación, resiliencia biomasa, conservación.

\section{REFERENCIAS}

Alvarado, J. J., \& Chiriboga, A. (2008). Distribución y abundancia de equinodermos en las aguas someras de la Isla del Coco, Costa Rica. Revista Biología Tropical, 56(Suplemento 2), 99-111. 
Alvarado, J. J., Cortés, J., Esquivel, M. F., \& Salas, E. (2012). Costa Rica's Marine ProtectedAreas: status and perspectives. Revista de Biología Tropical, 60, 129-142.

Alvarez-Filip, L., Côté, I. M., Gill, J. A., Watkinson, A. R., \& Dulvy, N. K. (2011). Region-wide temporal and spatial variation in Caribbean reef architecture: is coral cover the whole story? Global Change Biology, $17,2470-2477$

Bakus, G. J. (2007). Quantitative Analysis of Marine Biological Communities: Field Biology and Environment. New Jersey, EEUU: Wiley-Interscience.

Briones-Fourzán, P., \& Lozano-Álvarez, E. (2000). The spiny lobster fisheries in Mexico. En B. F. Phillips \& J. Kittaka (Eds.), Spiny Lobsters: Fisheries and Culture (pp. 169-188) Oxford, Reino Unido: Blackwell Science Ltd.

Clarke, K. R., \& Gorley, R. N. (2006). PRIMER v6: User Manual. Plymouth, U.K: PRIMER-E Ltd.

Clarke, K. R., \& Warwick, R. M. (2001). A further biodiversity index applicable to species lists: variation in taxonomic distinctness. Marine Ecology Progress Series, 216, 265-278.

Cortés, J. (2008a). Historia de la investigación marina de la Isla del Coco, Costa Rica. Revista de Biología Tropical, 56(Suplemento 2), 1-18.

Cortés, J. (Ed.). (2008b). Investigaciones marinas en la Isla del Coco, Costa Rica / Marine Research at Isla del Coco (Cocos Island), Costa Rica / Recherches marines à l'Ile du Coco, Costa Rica. Revista de Biología Tropical, 56(Suplemento 2), 1-217.

Cortes, J. (Ed.). (2012). Investigaciones marinas en el Parque Nacional Isla del Coco, Costa Rica. Revista de Biología Tropical, 60(Suplemento 3), 1-392.

Edgar, G. J., Banks, S., Fariña, J. M., Calvopiña, M., \& Martínez, C. (2004). Regional biogeography of shallow reef fish and macro-invertebrate communities in the Galapagos archipelago. Journal of Biogeography, $31,1107-1124$

Edgar, G. J., Banks, S. A., Bessudo, S., Cortés, J., Guzman, H. M., Henderson, S., Martinez, C., Rivera, F., Soler, G., Ruiz, D., \& Zapata, F. A. (2011). Variation in reef fish and invertebrate communities with level of protection from fishing across the Eastern Tropical Pacific seascape. Global Ecological Biogeography, 20,730-743

Edgar, G. J., Stuart-Smith, R. D., Willis, T. J., Kininmonth, S., Baker, S. C., Banks, S., Barrett, N. S., Becerro, M. A., Bernard, A. T. F., Berkhout, J., Buxton, C. D., Campbell, S. J., Cooper, A. T., Davey, M., Edgar, S. C., Försterra, G., Galván, D. E., Irigoyen, A. J., Kushner, D. J., Moura, R., Parnell, P. E., Shears, N. T., Soler, G., Strain, E. M. A., \& Thomson, R. J. (2014). Global conservation outcomes depend on marine protected areas with five key features. Nature, 506, 216-220.

Espinoza, M., Cappo, M., Heupel, M. R., Tobin, A. J., \& Simpfendorfer, C. A. (2014). Quantifying shark distribution patterns and species-habitat associations: implications of Marine Park zoning. PLoS ONE, 9, e106885.

Friedlander, A. M., \& DeMartini, E. E. (2002). Contrasts in density, size, and biomass of reef fishes between the northwestern and the main Hawaiian Islands: the effects of fishing down apex predators. Marine Ecology Progress Series, 230, 253-264.

Friedlander, A. M., Zgliczynski, B. J., Ballesteros, E., Aburto-Oropeza, O., Bolaños, A., \& Sala, E. (2012). The Shallow-water fish assemblage of Isla del Coco National Park, Costa Rica: structure and patterns in an isolated, predator-dominated ecosystem. Revista de Biología Tropical, 60(Suplemento 3), 321-338.

Glynn, P. W. (1974). The impact of Acanthaster on corals and coral reefs in the eastern Pacific. Environmental Conservation, 1, 295-304.

Glynn, P. W. (1997). Bioerosion and coral reef growth a dynamic balance. En C. Birkeland (Ed.), Life and Death on Coral Reefs (pp. 68-95). New York: Chapman and Hall.

Glynn, P. W., Enochs, I. C., Afflerbach, J. A., Brandtneris, V. W., \& Serafy, J. E. (2014). Eastern Pacific reef fish responses to coral recovery following El Niño disturbances. Marine Ecology Progress Series, 495, 233-247.

Graham, N. A. J., Jennings, S., MacNeil, M. A., Mouillot, D., \& Wilson, S. K. (2015). Predicting climate-driven regime shifts versus rebound potential in coral reefs. Nature, 518, 94-97.

Guzman, H. M., \& Cortés, J. (1992). Cocos Island (Pacific of Costa Rica) coral reefsafterthe 1982-83 El Niño disturbance. Revista de Biología Tropical, 40, 309-324.

Guzman, H. M., \& Cortés, J. (2007). Reef recovery 20-yr after the 1982-83 El Niño massive mortality. Marine Biology, 151, 401-411.

Guzman, H. M., Guevara, C. A., \& Breedy, O. (2004). Distribution, diversity, and conservation of coral reefs and coral communities in the largest marine protected area of Pacific Panama (Coiba Island). Enviromental Conservation, 31, 1-11

Guzman, H. M., Benfield, S., Breedy, O., \& Mair, J. M. (2008a). Broadening reef protection across the Marine Conservation Corridor of the Eastern Tropical Pacific: Distribution and diversity of reefs in Las Perlas Archipelago, Panama. Environmental Conservation, 35, 46-54.

Guzman, H. M., Cipriani, R., Vega, A. J., López, M., \& Mair, J. M. (2008b). Population assessment of the 
Pacific green spiny lobster Panulirus gracilis in Pacific Panama. Journal of Shellfish Research, 27, 907-915.

Lemare, M., Pecorino, D., Hardy, N., Liddy, M., Byrne, M., \& Uthicke, S. (2014). The thermal tolerance of crown-of-thorns (Acanthaster planci) embryos and bipinnaria larvae: implications for spatial and temporal variation in adult populations. Coral Reefs, 33, 207-219.

Lee, S. C. (2006). Habitat complexity and consumermediated positive feedbacks on a Caribbean coral reef. Oikos, 112, 442-447.

Lizano, O. G. (2008). Dinámica de aguas alrededor de la Isla del Coco, Costa Rica. Revista de Biología Tropical, 56(Suplemento 2): 31-48.

Lizano, O.G. 2012. Rasgos morfológicos alrededor de la Isla del Coco y de sus montes submarinos vecinos, Pacífico de Costa Rica. Revista de Biología Tropical, 60(Suplemento 3): 43-51.

McCauley, D. J, Pinsky, M. L., Palumbi, S. R., Estes, J. A., Joyce, F. H., \& Warner, R. R. (2015). Marine defaunation: animal loss in the global ocean. Science, 347, 248-253.

Moreno-Díaz, M. L. (2012). Actividades socioeconómicas en el Parque Nacional Isla del Coco, Costa Rica y posibles efectos de la variabilidad climática. Revista de Biología Tropical, 60(Suplemento 3), 113-129.

Palacios, M., \& Zapata, F. A. (2014). Fish community structure on coral habitats with contrasting architecture in the Tropical Eastern Pacific. Revista de Biología Tropical, 62(Suplemento 1), 343-357.

Palacios, M. M., Muñoz, C. G., \& Zapata, F. A. (2014). Fish corallivory on a pocilloporid reef and experimental coral responses to predation. Coral Reefs, 33, 625-636.

Purcell, S. W., Polidoro, B. A., Hamel, J. F., Gamboa, R. U., \& Mercier, A. (2014). The cost of being valuable: predictors of extinction risk in marine invertebrates exploited as luxury seafood. Proceedings of the Royal Society B-Biological Sciences, 281, 20133296.

Reyes-Bonilla, H., Herrero-Pérezrul, M. D., GonzálezRomero, S., González-Peralta, A., \& Ramírez-Hernández, Y. (2008). Abundance of the brown sea cucumber Isostichopus fuscus at the National Park Bahía de Loreto, México. Revista de Biología Tropical, 56(Suplemento 3), 265-271.

Rogers, C. S., Garrison, G., Grober, R., Hillis, Z. M., \& Franke, M. A. (1994). Manual para el monitoreo de arrecifes de coral en el Caribe y el Atlántico Occidental. Islas Vírgenes, EEUU: The Nature Conservancy, World Wild Fund.

Sandin, S. A., Smith, J. E., DeMartini, E. E., Dinsdale, E. A., Donner, S. D., Friedlander, A. M., Konotchick, T., Malay, M., Maragos, J. E., Obura, D., Pantos, O., Paulay, G., Richie, M., Rohwer, F., Schroeder, R. E., Walsh, S., Jackson, J. B. C., Knowlton, N., \& Sala, E. (2008). Degradation of coral reef communities across a gradient of human disturbance. PLOS ONE, 3, e1548.

Sibaja-Cordero, J. A. (2008). Tendencias espacio-temporales de los avistamientos de fauna marina en los buceos turísticos (Isla del Coco, Costa Rica). Revista de Biología Tropical, 56(Suplemento 2), 113-132.

Sibaja-Cordero, J. A., Troncoso, J. S., Benavides-Varela, C., \& Cortés, J. (2012). Distribution of shallow water soft and hard bottom seabeds in the Isla del Coco National Park, Pacific Costa Rica. Revista Biología Tropical, 60(Suplemento 3), 53-66.

UICN. (2001). The IUCN Red List of Theatened Species. Categories \& Criteria (version 3.1). Recuperado de http://www.iucnredlist.org/static/ categories_criteria_3_1

Vargas-Castillo, R., \& Wehrtmann I. S. (2008). Stomatopods and decapods from Isla del Coco, Pacific Costa Rica. Revista Biología Tropical, 56(Suplemento 2), 79-97.

Vargas-Castillo, R., \& Wehrtmann, I. S. (2009). Decapod Crustaceans. En I. S. Wehrtmann \& J. Cortés (Eds.). Marine Biodiversity of Costa Rica, Central America (pp. 209-228). New York: Springer.

Weinberg, S. (1981). A comparison of coral reef survey methods. Bijdragen tot de Dierkunde, 51, 199-218.

Weston-Knight, J. C. (1992). La Isla del Coco (Cocos Island). San José, Costa Rica: Trejos Hermanos Sucesores.

White, E. R., Myers, M. C., Mills-Flemming, J., \& Baum, J. K. (2015). Shifting elasmobranch community assemblage at Cocos Island - an isolated marine protected area. Conservation Biology, 29, 1186-1197.

Zanella, I., López-Garro, A., Golfín-Duarte, G., \& Sáenz, J. (2012), Abundancia, tamaño y estructura poblacional del tiburón punta blanca de arrecife, Triaenodon obesus (Carcharhiniformes: Carcharhinidae), en Bahía Chatham, Parque Nacional Isla del Coco, Costa Rica. Revista de Biología Tropical, 60(Suplemento 3), 339-346. 
